\title{
Semilinear Duhem Model for Rate-Independent and Rate-Dependent Hysteresis
}

\author{
JinHyoung Oh, Student Member, IEEE, and Dennis S. Bernstein, Fellow, IEEE
}

\begin{abstract}
The classical Duhem model provides a finite-dimensional differential model of hysteresis. In this paper, we consider rate-independent and rate-dependent semilinear Duhem models with provable properties. The vector field is given by the product of a function of the input rate and linear dynamics. If the input rate function is positively homogeneous, then the resulting input-output map of the model is rate independent, yielding persistent nontrivial input-output closed curve (that is, hysteresis) at arbitrarily low input frequency. If the input rate function is not positively homogeneous, the input-output map is rate dependent and can be approximated by a rate-independent model for low frequency inputs. Sufficient conditions for convergence to a limiting input-output map are developed for rate-independent and rate-dependent models. Finally, the reversal behavior and orientation of the rate-independent model are discussed.
\end{abstract}

Index Terms-Duhem, hysteresis, rate dependence.

\section{INTRODUCTION}

$\mathbf{H}$ YSTERESIS arises in diverse applications, such as structural mechanics, aerodynamics, and electromagnetics. The word "hysteresis" connotes lag, and hysteretic systems are generally described as having memory. Although there is no precise definition of hysteresis, we adopt the notion that hysteresis is effectively nontrivial quasi-dc input-output closed curve, that is, a nontrivial closed curve in the input-output map that persists for an periodic input as the frequency content of the input signal approaches dc. With dynamic, that is, non-dc excitation, both linear and nonlinear systems exhibit nontrivial input-output closed curve, which is generally frequency dependent and a natural consequence of the system's dynamics. However, the input-output map a linear system can approach only a single-valued linear map as the input frequency approaches zero. Consequently no linear system is hysteretic, and thus hysteresis is an inherently nonlinear phenomenon.

To illustrate this point of view, consider the mechanism [1] shown in Fig. 1, where the system input $r(t)$ is the position of the right end of the spring and the system output $q(t)$ is the position of the mass. The equation of motion is given by

$$
m \ddot{q}(t)+c \dot{q}(t)+k d_{w}(q(t)-r(t))=0
$$

where $d_{w}(z)$ is a deadzone function with width $w$ due to the gap where the spring attaches to the mass. The presence of hysteresis

Manuscript received May 27, 2004; revised October 2, 2004. Recommended by Associate Editor M. Reyhanoglu. This work was supported in part by the National Science Foundation under Grant ECS-0225799.

The authors are with the Department of Aerospace Engineering, The University of Michigan, Ann Arbor, MI 48109-2140 USA (e-mail: johzz@umich.edu; dsbaero@umich.edu).

Digital Object Identifier 10.1109/TAC.2005.847035

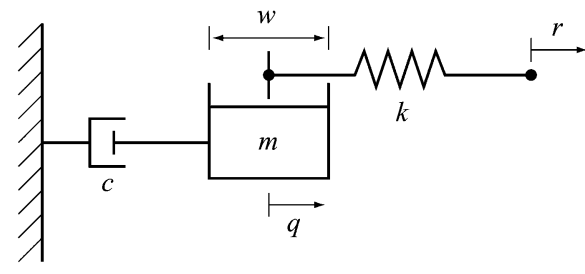

Fig. 1. Mass-dashpot-spring system with gap.
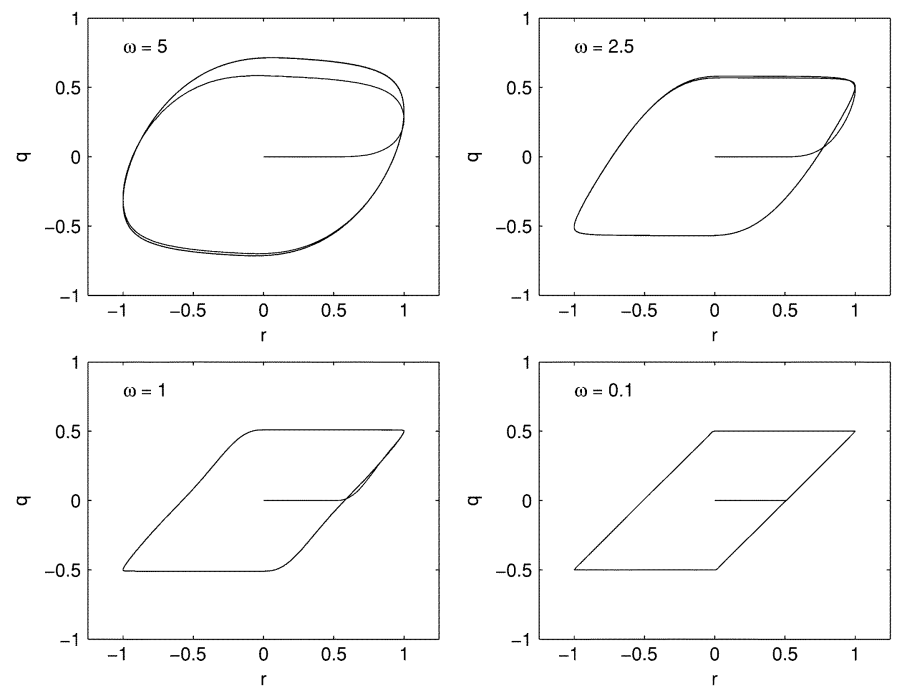

Fig. 2. Input-output map for the mass-dashpot-spring system with gap $w=1$ when $m=0.1, k=10, c=1$, and $r(t)=\sin \omega t$.

between $r(t)$ and $q(t)$ is not readily evident during dynamic operation, since the nontrivial input-output closed curve is a consequence of both the gap and the dynamics. However, Fig. 2 reveals that the nontrivial closed curve persists near dc, that is, in the dc limit at asymptotically low frequency.

Alternatively, consider the relationship between the magnetic field strength $H(t)$ and the magnetic flux density $B(t)$ of a ferromagnetically soft material of the isoperm type [2] given by

$$
\dot{B}(t)=a|\dot{H}(t)|[b H(t)-B(t)]+c \dot{H}(t)
$$

where $a, b, c$ are positive constants. Fig. 3 shows the relationship between the magnetic field strength $H(t)$ and the magnetic flux density $B(t)$. The presence of nontrivial input-output closed curve for low frequency inputs indicates that this system is hysteretic.

Although the examples discussed above are both hysteretic, they are distinct in several respects. First, the shapes of the hysteresis maps are different, although their orientation is the same, namely, counterclockwise. Next, the response of the 

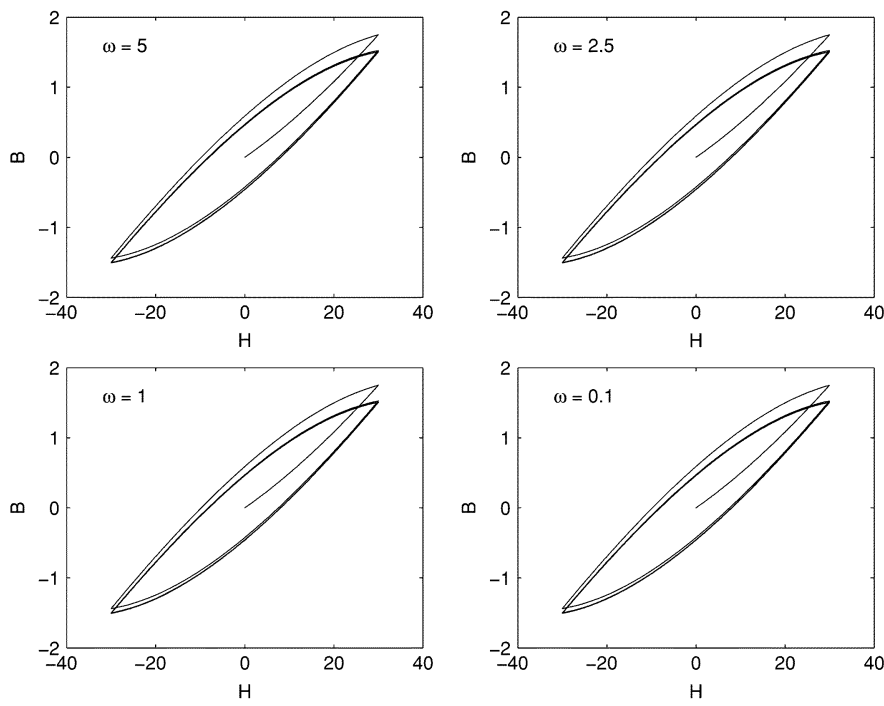

Fig. 3. Input-output map for a ferromagnetically soft material of the isoperm type when $a=0.02125, b=0.100, c=0.04361$, and $H(t)=30 \sin \omega t$.

mass-spring system depends on the input frequency, whereas the ferromagnetic material model has the same input-output response for all input frequencies.

In classical terminology [2]-[4], these systems have ratedependent hysteresis and rate-independent hysteresis, respectively. We shall use this traditional terminology despite the fact that it is inconsistent with our view that "hysteresis" per se refers to the response in the dc limit. Since the dynamic response of a system with rate-independent hysteresis is completely determined by its quasi-dc behavior, such a system is effectively kinematic.

Examples (1.1) and (1.2) are also different with respect to their dependence on the shape of the input. Consider the input-output maps corresponding to sinusoidal and triangle-wave inputs. Fig. 4 shows that the mass-spring example exhibits different input-output maps, while Fig. 5 shows that the ferromagnetic material model exhibits the same maps. This distinction suggests a relationship between the rate dependence of the hysteresis and the dependence of the input-output map on the shape of the periodic input.

Yet another distinction between these examples is their reversal behavior. As can be seen in Fig. 6(a), the mass-spring example exhibits play-type reversal, while the ferromagnetic material model response converges to a self-similar hysteresis as seen in Fig. 6(b).

One of the most popular hysteresis models is the infinite-dimensional Preisach model [4]-[9], which is an integral model whose kernel involves an infinite number of hysteretic operators. The Preisach model can capture a large class of hysteresis maps with complex reversal behavior. However, simulating Preisach models requires gridding of the plane and thus is computationally demanding. Other integral models of hysteresis with different kernel functions include the energy-based hysteresis model [10], the generalized Maxwell-slip hysteretic friction model [11], [12], and the Prandtl-Ishilinskii model [13, pp. 342-367].

In contrast to Preisach models, the examples discussed above are finite dimensional. The mass-spring example is suggested

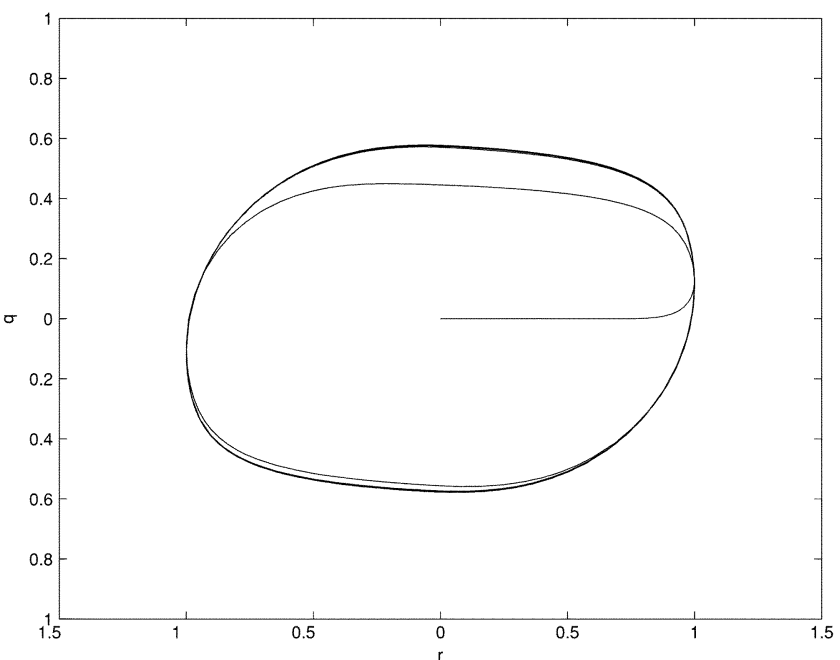

(a)

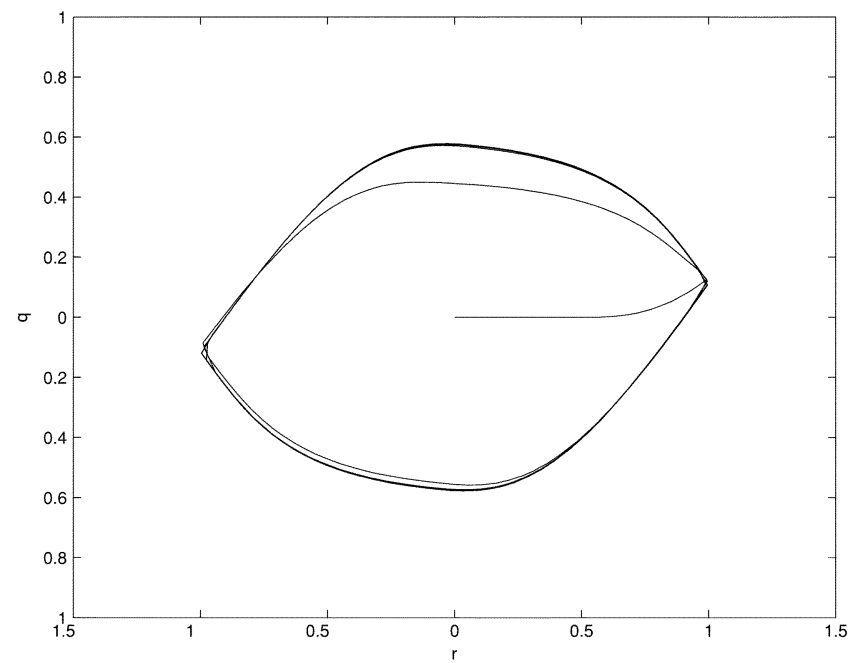

(b)

Fig. 4. Input-output map for the mass-dashpot-spring system with deadzone under (a) sinusoid input and (b) triangle-wave input when $r=\sin 5 t$.

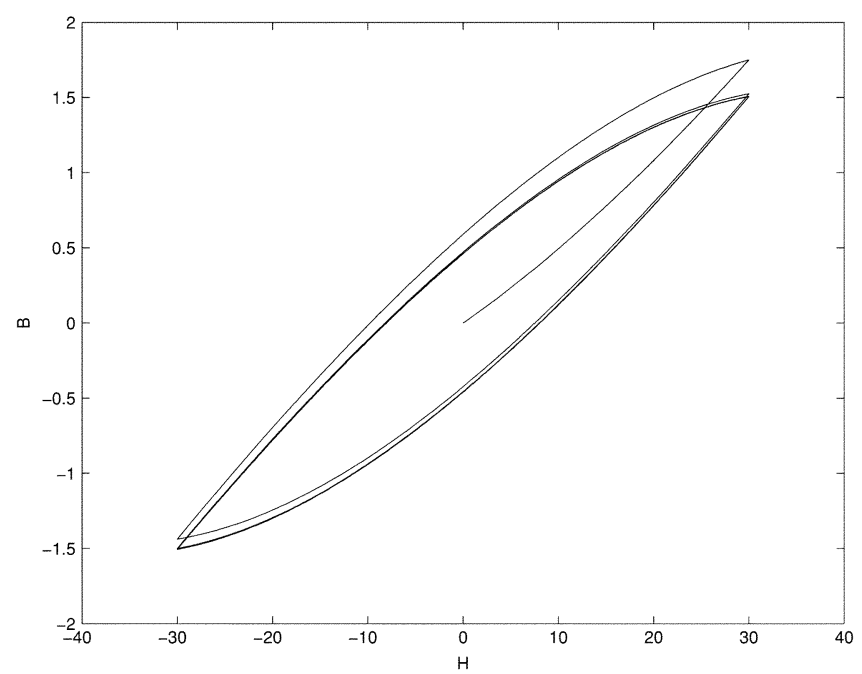

Fig. 5. Input-output map for the model of a ferromagnetically soft material of the isoperm type under both sinusoidal and triangle-wave inputs.

in $[13$, p. 90$]$ as an approximation to a hysteron model, while the ferromagnetic material model is a Duhem model, a class of hysteresis models studied in [2] and [14]-[16]. Variations of 


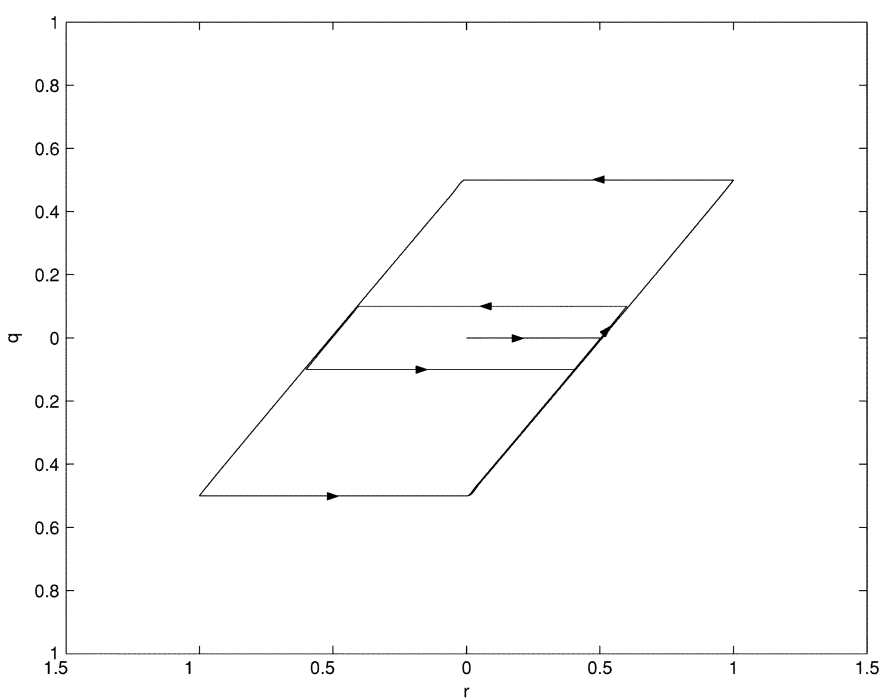

(a)

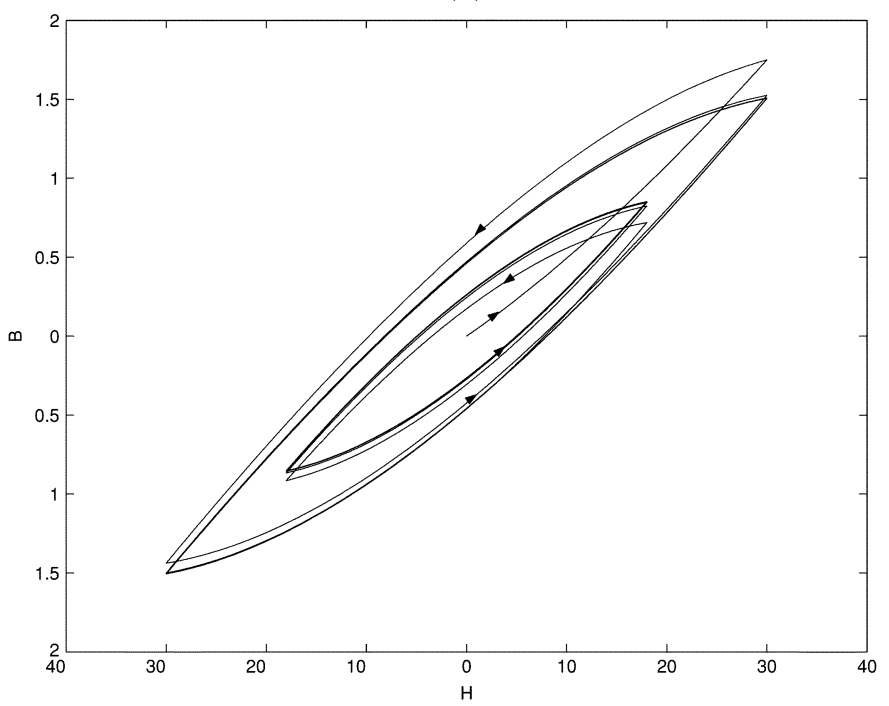

(b)

Fig. 6. Reversal behavior for (a) the mass-dashpot-spring system with deadzone and (b) the ferromagnetically soft material.

the Duhem model have been studied in different contexts under various names. For example, the Bouc-Wen model [17], [18], the Madelung model [13, p. 274], the Dahl friction model [19], the LuGre friction model [20], and the presliding friction model [21] are specialized Duhem models as shown in Examples 2.1 and 2.2. All of these models are rate independent. Rate-dependent models are studied in [3] and [14].

The purpose of this paper is to extend the existing analysis of the Duhem model to further elucidate its properties. In particular we are interested in the rate dependence of the response, convergence, shape, and orientation of the hysteresis map, as well as the reversal behavior.

The contents of the paper are as follows. In Section II, we introduce the generalized Duhem model and define the hysteresis map to be the dc-limiting input-output phase portrait. In Section III, we discuss the rate-independent generalized Duhem model using the concept of positive time-scale invariance. In Sections IV and V we introduce the rate-independent and rate- dependent semilinear Duhem models, respectively, and analyze their properties. In Section VI, the reversal behavior of the rateindependent generalized Duhem model is discussed. Finally, the orientation of the limiting periodic input-output map of the rate-independent generalized Duhem model is analyzed in Section VII, followed by the Conclusion.

\section{GENERALIZED DUHEM MODEL}

Consider the single-input single-output generalized Duhem model given by

$$
\begin{aligned}
& \dot{x}(t)=f(x(t), u(t)) g(\dot{u}(t)), \quad x(0)=x_{0}, \quad t \geq 0 \\
& y(t)=h(x(t), u(t))
\end{aligned}
$$

where $x:[0, \infty) \rightarrow \mathbb{R}^{n}$ is absolutely continuous, $u:[0, \infty) \rightarrow$ $\mathbb{R}$ is continuous and piecewise $C^{1}, f: \mathbb{R}^{n} \times \mathbb{R} \rightarrow \mathbb{R}^{n \times r}$ is continuous, $g: \mathbb{R} \rightarrow \mathbb{R}^{r}$ is continuous and satisfies $g(0)=0, y$ : $[0, \infty) \rightarrow \mathbb{R}$, and $h: \mathbb{R}^{n} \times \mathbb{R} \rightarrow \mathbb{R}$ is continuous. The value of $\dot{x}(t)$ at a point $t$ at which $\dot{u}(t)$ is discontinuous can be assigned arbitrarily. We assume that the solution to (2.1) exists and is unique on all finite intervals. Under these assumptions, $x$ and $y$ are continuous and piecewise $C^{1}$. The following definition will be useful.

Definition 2.1: The nonempty set $\mathcal{H} \subset \mathbb{R}^{2}$ is a closed curve if there exists a continuous, piecewise $C^{1}$, and periodic map $\gamma:[0, \infty) \rightarrow \mathbb{R}^{2}$ such that $\gamma([0, \infty))=\mathcal{H}$.

Definition 2.1 implies that every closed curve is a compact and connected subset of $\mathbb{R}^{2}$. For closed curves $\mathcal{H}_{1}, \mathcal{H}_{2}$, define the Hausdorff metric [22, p. 104]

$$
\begin{array}{r}
d\left(\mathcal{H}_{1}, \mathcal{H}_{2}\right) \triangleq \max \left\{\sup _{\eta_{1} \in \mathcal{H}_{1}}\left(\inf _{\eta_{2} \in \mathcal{H}_{2}}\left\|\eta_{1}-\eta_{2}\right\|\right),\right. \\
\left.\sup _{\eta_{2} \in \mathcal{H}_{2}}\left(\inf _{\eta_{1} \in \mathcal{H}_{1}}\left\|\eta_{1}-\eta_{2}\right\|\right)\right\}
\end{array}
$$

where $\|\cdot\|$ is a norm on $\mathbb{R}^{2}$. Since $\mathbb{R}^{2}$ with the metric $d(x, y) \triangleq$ $\|x-y\|$ is complete, it follows from [23, p. 67] that the set of closed curves with $d(\cdot, \cdot)$ is a complete metric space.

Definition 2.2: Let $u:[0, \infty) \rightarrow\left[u_{\min }, u_{\max }\right]$ be continuous, piecewise $C^{1}$, periodic with period $\alpha$, and have exactly one local maximum $u_{\max }$ in $[0, \alpha)$ and exactly one local minimum $u_{\min }$ in $[0, \alpha)$. For all $T>0$, define $u_{T}(t) \triangleq u(\alpha t / T)$, assume that there exists $x_{T}:[0, \infty) \rightarrow \mathbb{R}^{n}$ that is periodic with period $T$ and satisfies (2.1) with $u=u_{T}$, and let $y_{T}$ : $[0, \infty) \rightarrow \mathbb{R}$ be given by (2.2) with $x=x_{T}$ and $u=u_{T}$. For all $T>0$, the periodic input-output map $\mathcal{H}_{T}\left(u_{T}, y_{T}\right)$ is the closed curve $\mathcal{H}_{T}\left(u_{T}, y_{T}\right) \triangleq\left\{\left(u_{T}(t), y_{T}(t)\right): t \in[0, \infty)\right\}$, and the limiting periodic input-output map $\mathcal{H}_{\infty}(u)$ is the closed curve $\mathcal{H}_{\infty}(u) \triangleq \lim _{T \rightarrow \infty} \mathcal{H}_{T}\left(u_{T}, y_{T}\right)$ if the limit exists. If there exist $\left(u, y_{1}\right),\left(u, y_{2}\right) \in \mathcal{H}_{\infty}(u)$ such that $y_{1} \neq y_{2}$, then $\mathcal{H}_{\infty}(u)$ is a hysteresis map, and the generalized Duhem model is hysteretic.

Example 2.1: Bouc's hysteresis model [17] is given by

$$
\begin{aligned}
y(t)=\mu u(t)+h_{0}( & u(t)) \\
& +\int_{0}^{t} F\left(\int_{\tau}^{t}|\dot{u}(\sigma)| \mathrm{d} \sigma\right) \mathrm{d} \Phi(u(\tau))
\end{aligned}
$$


where $\mu \geq 0, h_{0}: \mathbb{R} \rightarrow \mathbb{R}$ and $F: \mathbb{R} \rightarrow \mathbb{R}$ are continuous, and $\Phi: \mathbb{R} \rightarrow \mathbb{R}$ is $C^{1}$. Letting $F(v)=e^{-\gamma v}$, where $\gamma>0$, it follows that (2.4) is equivalent to

$$
\begin{aligned}
& \dot{x}(t)=-\gamma|\dot{u}(t)| x(t)+\frac{\mathrm{d} \Phi(u(t))}{\mathrm{d} u(t)} \dot{u}(t) \\
& y(t)=x(t)+\mu u(t)+h_{0}(u(t)) .
\end{aligned}
$$

Now, (2.5) and (2.6) can be rewritten in the form of (2.1) and (2.2) as

$$
\begin{aligned}
& \dot{x}(t)=\left[\begin{array}{ll}
-\gamma x(t)+\frac{\mathrm{d} \Phi(u(t))}{\mathrm{d} u(t)} & \gamma x(t)+\frac{\mathrm{d} \Phi(u(t))}{\mathrm{d} u(t)}
\end{array}\right]\left[\begin{array}{c}
\dot{u}_{+}(t) \\
\dot{u}_{-}(t)
\end{array}\right] \\
& y(t)=x(t)+\mu u(t)+h_{0}(u(t))
\end{aligned}
$$

where

$$
\dot{u}_{+}(t) \triangleq \max \{0, \dot{u}(t)\} \quad \dot{u}_{-}(t) \triangleq \min \{0, \dot{u}(t)\} .
$$

The Bouc-Wen model [18] is the variation of (2.7) and (2.8) given by (2.10) and (2.11), shown at the bottom of the page, where $\beta, k$, and $d$ are system parameters, and $y(t)$ is the restoring force of a hysteretic structural system due to a displacement $u(t)$. Setting $\beta=-1, k=1$, and $d=0$ yields the Dahl friction model [19]

$$
\dot{x}(t)=\left[\begin{array}{ll}
-\gamma x(t)+1 & \gamma x(t)+1
\end{array}\right]\left[\begin{array}{l}
\dot{u}_{+}(t) \\
\dot{u}_{-}(t)
\end{array}\right]
$$

where $u(t)$ denotes rigid body displacement and $x(t)$ is the elastic strain in the frictional contact.

Example 2.2: LuGre friction model [20] is given by

$$
\begin{aligned}
\dot{x}(t) & =\dot{u}-\frac{\dot{u}(t)}{r(\dot{u}(t))} x(t) \\
y(t) & =\sigma_{0} x(t)+\sigma_{1} \dot{x}(t)+\sigma_{2} \dot{u}(t)
\end{aligned}
$$

where $r: \mathbb{R} \rightarrow \mathbb{R}$ is monotonically decreasing, $u$ denotes the relative position between the two surfaces, $y$ denotes the friction force, and $\sigma_{0}, \sigma_{1}, \sigma_{2}>0$ are stiffness, damping coefficient, and viscous friction coefficient, respectively. The state equation (2.13) can be rewritten as

$$
\dot{x}(t)=\left[\begin{array}{ll}
1 & x(t)
\end{array}\right]\left[\begin{array}{c}
\dot{u}(t) \\
-\frac{|\dot{u}(t)|}{r(\dot{u}(t))}
\end{array}\right]
$$

which is in the form of (2.1). Note that (2.14) depends on $\dot{u}(t)$ and thus cannot be written as (2.2). This model can be interpreted as an improper generalized Duhem model. Other im- proper generalized Duhem models include the presliding friction model [21]

$$
\begin{aligned}
& \dot{x}(t)=\dot{u}(t)\left(1-\operatorname{sgn}\left(\frac{F_{\mathrm{d}}(x(t))}{S(\dot{u}(t))-F_{\mathrm{b}}}\right)\left|\frac{F_{\mathrm{d}}(x(t))}{S(\dot{u}(t))-F_{\mathrm{b}}}\right|^{k}\right) \\
& y(t)=F_{\mathrm{b}}+F_{\mathrm{d}}(x(t))+\sigma_{1} \dot{x}(t)+\sigma_{2} \dot{u}(t)
\end{aligned}
$$

where $F_{\mathrm{d}}: \mathbb{R} \rightarrow \mathbb{R}$ is the current transition curve, $S: \mathbb{R} \rightarrow \mathbb{R}$ models the constant velocity behavior in sliding, $F_{\mathrm{b}} \in \mathbb{R}$ is an offset constant, and $k$ is a system parameter. The state equation (2.16) can be written in the form of (2.1) as

$$
\begin{aligned}
& \dot{x}(t)=\left[\begin{array}{ll}
1 & \operatorname{sgn}\left(F_{\mathrm{d}}(x(t))\right)\left|F_{\mathrm{d}}(x(t))\right|^{k}
\end{array}\right] \\
& \times\left[\begin{array}{c}
\dot{u}(t) \\
\left.-\operatorname{sgn}\left(\frac{\dot{u}(t)}{S(\dot{u}(t))-F_{\mathrm{b}}}\right)\left|\frac{\dot{u}(t)}{S(\dot{u}(t))-F_{\mathrm{b}}}\right|^{k}\right] .
\end{array}\right.
\end{aligned}
$$

\section{RATE-INDEPENDENT GENERALIZED DUHEM ModeL}

In this section, we consider the rate-independent generalized Duhem model. The following definition is needed.

Definition 3.1: The continuous and piecewise $C^{1}$ function $\tau:[0, \infty) \rightarrow[0, \infty)$ is a positive time scale if $\tau(0)=0, \tau$ is nondecreasing, and $\lim _{t \rightarrow \infty} \tau(t)=\infty$. The generalized Duhem model (2.1), (2.2) is rate independent if, for every continuous and piecewise $C^{1} x$ and $u$ satisfying (2.1) and for every positive time scale $\tau$, it follows that $x_{\tau}(t) \triangleq x(\tau(t))$ and $u_{\tau}(t) \triangleq$ $u(\tau(t))$ also satisfy (2.1).

Note that there is a slight abuse of notation in defining $x_{T}, u_{T}$ in Definition 2.2, and $x_{\tau}, u_{\tau}$ in Definition 3.1. Letting $\tau(t)=$ $\alpha t / T$, it follows that $x_{\tau}$ and $u_{\tau}$ denote $x_{T}$ and $u_{T}$, respectively. The following definition is needed to characterize the rate-independent generalized Duhem model.

Definition 3.2: The function $g$ is positively homogeneous if $g(h v)=h g(v)$ for all $h \geq 0$ and $v \in \mathbb{R}$.

The following result generalizes [14, Prop. 9].

Proposition 3.1: Assume that $g$ is positively homogeneous. Then, the generalized Duhem model (2.1), (2.2) is rate independent.

Proof: Let $\tau$ be a positive time scale. Then $\tau(0)=0$ and, thus, $x_{\tau}(0)=x(\tau(0))=x(0)=x_{0}$. Now, for all $t>0$, consider

$$
\begin{aligned}
& \frac{\mathrm{d} x_{\tau}(t)}{\mathrm{d} t}-f\left(x_{\tau}(t), u_{\tau}(t)\right) g\left(\frac{\mathrm{d} u_{\tau}(t)}{\mathrm{d} t}\right) \\
& \quad=\frac{\mathrm{d} x(\tau(t))}{\mathrm{d} t}-f(x(\tau(t)), u(\tau(t))) g\left(\frac{\mathrm{d} u(\tau(t))}{\mathrm{d} t}\right) \\
& \quad=\dot{\tau}(t) \frac{\mathrm{d} x(\tau)}{\mathrm{d} \tau}-f(x(\tau), u(\tau)) g\left(\dot{\tau}(t) \frac{\mathrm{d} u(\tau)}{\mathrm{d} \tau}\right) .
\end{aligned}
$$

$$
\begin{aligned}
\dot{x}(t) & =\left[-\gamma x(t)|x(t)|^{k-1}-\beta|x(t)|^{k}, \quad \gamma x(t)|x(t)|^{k-1}-\beta|x(t)|^{k}\right]\left[\begin{array}{c}
\dot{u}_{+}(t) \\
\dot{u}_{-}(t)
\end{array}\right] \\
y(t) & =x(t)+d u(t)
\end{aligned}
$$


Since $\tau$ is a positive time scale, $\dot{\tau}(t) \geq 0$ for all $t \geq 0$. Hence, it follows from the positive homogeneity of $g$ that

$$
g\left(\dot{\tau}(t) \frac{\mathrm{d} u_{\tau}(t)}{\mathrm{d} t}\right)=\dot{\tau}(t) g\left(\frac{\mathrm{d} u_{\tau}(t)}{\mathrm{d} t}\right)
$$

which implies that

$$
\begin{aligned}
& \frac{\mathrm{d} x_{\tau}(t)}{\mathrm{d} t}-f\left(x_{\tau}(t), u_{\tau}(t)\right) g\left(\frac{\mathrm{d} u_{\tau}(t)}{\mathrm{d} t}\right) \\
& \quad=\dot{\tau}(t)\left[\frac{\mathrm{d} x(\tau)}{\mathrm{d} \tau}-f(x(\tau), u(\tau)) g\left(\frac{\mathrm{d} u(\tau)}{\mathrm{d} \tau}\right)\right] \\
& \quad=0
\end{aligned}
$$

for all $t>0$, as required.

Let $\tau$ be a positive time scale, assume that (2.1) and (2.2) are rate independent, let $u(t)$ and $u_{\tau}(t)$, where $t \geq 0$, be inputs as in Definition 3.1, and let $x(t), y(t)$, and $x_{\tau}(t), y_{\tau}(t)$ satisfy (2.1), (2.2) with $u(t)$ and $u_{\tau}(t)$, respectively. Define the input-output map

$$
\mathcal{F}(u, y) \triangleq\{(u(t), y(t)): t \in[0, \infty)\}
$$

and the scaled input-output map

$$
\mathcal{F}_{\tau}\left(u_{\tau}, y_{\tau}\right) \triangleq\left\{\left(u_{\tau}(t), y_{\tau}(t)\right): t \in[0, \infty)\right\} .
$$

Then

$$
\begin{aligned}
\mathcal{F}_{\tau}\left(u_{\tau}, y_{\tau}\right) & =\left\{\left(u_{\tau}(t), h\left(x_{\tau}(t), u_{\tau}(t)\right)\right): t \in[0, \infty)\right\} \\
& =\{(u(\tau(t)), h(x(\tau(t)), u(\tau(t)))): t \in[0, \infty)\} \\
& =\{(u(\tau(t)), y(\tau(t))): t \in[0, \infty)\} \\
& =\{(u(t), y(t)): t \in[0, \infty)\} \\
& =\mathcal{F}(u, y) .
\end{aligned}
$$

Hence, $\mathcal{F}_{\tau}\left(u_{\tau}, y_{\tau}\right)$ is independent of $\tau$, and thus $\mathcal{F}_{\tau}\left(u_{\tau}, y_{\tau}\right)=$ $\mathcal{F}(u, y)$ for every positive time-scale $\tau$.

This observation shows that the periodic input-output map of the rate-independent generalized Duhem model is identical to the limiting periodic input-output map. Specifically, since $\tau(t)=\alpha t / T$ is a positive time scale, the periodic input-output map $\mathcal{H}_{T}\left(u_{T}, y_{T}\right)$ is invariant for all $T>0$ and, thus, $\mathcal{H}_{\infty}(u)=$ $\lim _{T_{0} \rightarrow \infty} \mathcal{H}_{T_{0}}\left(u_{T_{0}}, y_{T_{0}}\right)=\mathcal{H}_{T}\left(u_{T}, y_{T}\right)$, for all $T>0$.

The rate-independent generalized Duhem model has several alternative representations. The following lemma is needed for further discussion.

Lemma 3.1: Assume $g$ is positively homogeneous. Then, there exist $h_{+}, h_{-} \in \mathbb{R}^{r}$ such that

$$
g(v)= \begin{cases}v h_{+}, & v \geq 0 \\ v h_{-}, & v<0 .\end{cases}
$$

Proof: Let $g=\left[\begin{array}{lll}g_{1} & \cdots & g_{r}\end{array}\right]^{\mathrm{T}}$. Since $g$ is positively homogeneous, $g_{i}$ is also positively homogeneous, $i=1, \ldots, r$. Then, for all $v>0, g_{i}(v)=v g_{i}(1)=v h_{i_{+}}$, where $h_{i_{+}} \triangleq g_{i}(1), i=$ $1, \ldots, r$. Similarly, for all $v<0, g_{i}(v)=-v g_{i}(-1)=v h_{i_{-}}$, where $h_{i_{-}} \triangleq-g_{i}(-1), i=1, \ldots, r$. Finally, define $h_{+} \triangleq$ $\left[\begin{array}{lll}h_{1_{+}} & \cdots & h_{r_{+}}\end{array}\right]^{\mathrm{T}}$ and $h_{-} \triangleq\left[\begin{array}{lll}h_{1_{-}} & \cdots & h_{r_{-}}\end{array}\right]^{\mathrm{T}}$, which satisfies (3.1) as required.

Assume $g$ is positively homogeneous. Then, (2.1) and (2.2) can be written in the form of the Madelung model [13, p. 282]

$$
\begin{aligned}
& \dot{x}(t)= \begin{cases}f_{+}(x(t), u(t)) \dot{u}(t), & \dot{u}(t) \geq 0 \\
f_{-}(x(t), u(t)) \dot{u}(t), & \dot{u}(t)<0\end{cases} \\
& y(t)=h(x(t), u(t)), \quad x(0)=x_{0}, t \geq 0
\end{aligned}
$$

where $f_{+}(x(t), u(t)) \triangleq f(x(t), u(t)) h_{+}$and $f_{-}(x(t), u(t)) \triangleq$ $f(x(t), u(t)) h_{-}$. Note that (3.2) can be viewed as a switching system with respect to the sign of $\dot{u}(t)$. Furthermore, (3.1) can be written as

$$
g(\dot{u}(t))=h_{+} \dot{u}_{+}(t)+h_{-} \dot{u}_{-}(t)=\left[h_{+} \quad h_{-}\right]\left[\begin{array}{l}
\dot{u}_{+}(t) \\
\dot{u}_{-}(t)
\end{array}\right]
$$

and, thus, (3.2) can be written as (2.1) with $r=2$ in the form

$$
\dot{x}(t)=\left[f_{+}(x(t), u(t)) \quad f_{-}(x(t), u(t))\right]\left[\begin{array}{l}
\dot{u}_{+}(t) \\
\dot{u}_{-}(t)
\end{array}\right]
$$

for $x(0)=x_{0}, \quad t \geq 0$, which is the standard Duhem model [15, p. 131]. Finally, it follows from (3.5) that

$$
\begin{aligned}
\dot{x}(t) & =\operatorname{vec}\left(\left[f_{+}(x(t), u(t)) \quad f_{-}(x(t), u(t))\right]\left[\begin{array}{l}
\dot{u}_{+}(t) \\
\dot{u}_{-}(t)
\end{array}\right]\right) \\
=\left(\left[\begin{array}{l}
\dot{u}_{+}(t) \\
\dot{u}_{-}(t)
\end{array}\right]^{\mathrm{T}} \otimes I_{n}\right) \operatorname{vec}\left[f_{+}(x(t), u(t))\right. & \left.f_{-}(x(t), u(t))\right]
\end{aligned}
$$

where vec is the column-stacking operator and $\otimes$ denotes the Kronecker product. Hence, the rate-independent generalized Duhem model (2.1), (2.2) is equivalent to

$$
\begin{aligned}
\dot{x}(t) & =\left[\begin{array}{ll}
\dot{u}_{+}(t) I_{n} & \dot{u}_{-}(t) I_{n}
\end{array}\right]\left[\begin{array}{l}
f_{+}(x(t), u(t)) \\
f_{-}(x(t), u(t))
\end{array}\right] \\
y(t) & =h(x(t), u(t)), \quad x(0)=x_{0}, t \geq 0 .
\end{aligned}
$$

The following result is needed to analyze the rate-independent generalized Duhem model (3.2), (3.3).

Proposition 3.2: Assume that $g$ is positively homogeneous and given by (3.1), where $h_{+}, h_{-} \in \mathbb{R}^{r}$, and let $\hat{x}:\left[u_{\min }, u_{\max }\right] \rightarrow \mathbb{R}^{n}$ and $\hat{y}:\left[u_{\min }, u_{\max }\right] \rightarrow \mathbb{R}$ satisfy

$$
\begin{aligned}
\frac{\mathrm{d} \hat{x}(u)}{\mathrm{d} u} & = \begin{cases}f_{+}(\hat{x}(u), u), & \text { when } u \text { increases } \\
f_{-}(\hat{x}(u), u), & \text { when } u \text { decreases } \\
0, & \text { otherwise }\end{cases} \\
\hat{y}(u) & =h(\hat{x}(u), u)
\end{aligned}
$$

for $u \in\left[u_{\min }, u_{\max }\right]$ and with initial condition $\hat{x}\left(u_{0}\right)=x_{0}$, where $f_{+}(x, u) \triangleq f(x, u) h_{+}, f_{-}(x, u) \triangleq f(x, u) h_{-}$, and $u_{0} \in\left[u_{\min }, u_{\max }\right]$. Furthermore, let $u:[0, \infty) \rightarrow\left[u_{\min }, u_{\max }\right]$ be piecewise monotonic, continuous, piecewise $C^{1}$, and $u(0)=u_{0}$. Then, $x(t) \triangleq \hat{x}(u(t))$ and $y(t) \triangleq \hat{y}(u(t))$ satisfy (3.2) and (3.3). 
Proof: For $t \geq 0$, note that

$$
\begin{aligned}
\frac{\mathrm{d} x(t)}{\mathrm{d} t} & =\frac{\mathrm{d} \hat{x}(u(t))}{\mathrm{d} t} \\
& =\frac{\mathrm{d} \hat{x}(u(t))}{\mathrm{d} u(t)} \frac{\mathrm{d} u(t)}{\mathrm{d} t} \\
& = \begin{cases}f_{+}(\hat{x}(u(t)), u(t)) \dot{u}(t), & \text { when } u(t) \text { increases } \\
f_{-}(\hat{x}(u(t)), u(t)) \dot{u}(t), & \text { when } u(t) \text { decreases } \\
0, & \text { otherwise }\end{cases} \\
& = \begin{cases}f_{+}(\hat{x}(u(t)), u(t)) \dot{u}(t), & \dot{u}(t) \geq 0 \\
f_{-}(\hat{x}(u(t)), u(t)) \dot{u}(t), & \dot{u}(t)<0\end{cases} \\
& =f(x(t), u(t)) g(\dot{u}(t))
\end{aligned}
$$

with the initial condition $\hat{x}\left(u_{0}\right)=\hat{x}(u(0))=x(0)=x_{0}$. Hence, $x(t)$ satisfies (3.2). Therefore, for all $t \geq 0, x(t)=$ $\hat{x}(u(t))$ and, thus, $y(t)=\hat{y}(u(t))$ from (3.3).

Proposition 3.2 shows that the rate-independent generalized Duhem model (3.2), (3.3) can be reparameterized with $u$ as the independent variable. Note that (3.8) and (3.9) can be viewed as a time-varying dynamical system with nonmonotonic time $u$. Hence, reparameterization of the rate-independent generalized Duhem model implies that the input-output map of $u(t)$ and $y(t)$ consists of a positive orbit arising from $f_{+}(\hat{x}, u)$ and a negative orbit arising from $f_{-}(\hat{x}, u)$. We exclude pathological inputs by assuming that $u(t)$ is piecewise monotonic.

Assume that the forward-time and backward-time solutions of (3.8) exist and are unique on all finite intervals. If $f_{+}=f_{-}$, then the positive and negative orbits of (3.8) are identical and thus the limiting periodic input-output map is not hysteretic. Therefore, the existence of hysteresis requires that $f_{+}(\hat{x}(u), u)$ and $f_{-}(\hat{x}(u), u)$ be different.

Example 3.1: Let $u(t)$ be the triangle wave

$$
u(t) \triangleq \begin{cases}t-4 q-1, & 4 q \leq t<4 q+2 \\ -t+4 q+3, & 4 q+2 \leq 4 q+4\end{cases}
$$

for $q=0,1,2, \ldots$, and consider the positive time scales

$$
\begin{aligned}
& \tau_{1}(t) \triangleq 2 t \\
& \tau_{2}(t) \triangleq t / 2 \\
& \tau_{3}(t) \triangleq \begin{cases}\sin \left(\frac{\pi}{2} t-\frac{\pi}{2}\right)+4 q+1, \quad 4 q \leq t<4 q+2 \\
-\sin \left(\frac{\pi}{2} t-\frac{\pi}{2}\right)+4 q+3, \quad 4 q+2 \leq t<4 q+4\end{cases} \\
& \tau_{4}(t) \triangleq \begin{cases}\frac{2}{3} t+\frac{4}{3} q, & 4 q \leq t<4 q+3 \\
2 t-4 q-4, & 4 q+3 \leq t<4 q+4\end{cases}
\end{aligned}
$$

for $q=0,1,2, \ldots$, and $t \geq 0$. Fig. 7 shows the triangle wave $u$ and the positive time-scaled triangle waves $u_{\tau_{1}}, u_{\tau_{2}}, u_{\tau_{3}}$, and $u_{\tau_{4}}$. Note that $\tau_{1}$ and $\tau_{2}$ change the period of $u$, while $\tau_{3}$ and $\tau_{4}$ change the shape of $u$. Now, consider the generalized Duhem model

$$
\begin{aligned}
& \dot{x}(t)=(-x(t)+u(t)) g(\dot{u}(t)) \\
& y(t)=x(t), \quad x(0)=0, t \geq 0
\end{aligned}
$$

and let $g(\dot{u})=|\dot{u}|$. Since $g$ is positively homogeneous, Proposition 3.1 implies that (3.10), (3.11) is rate independent. Fig. 8
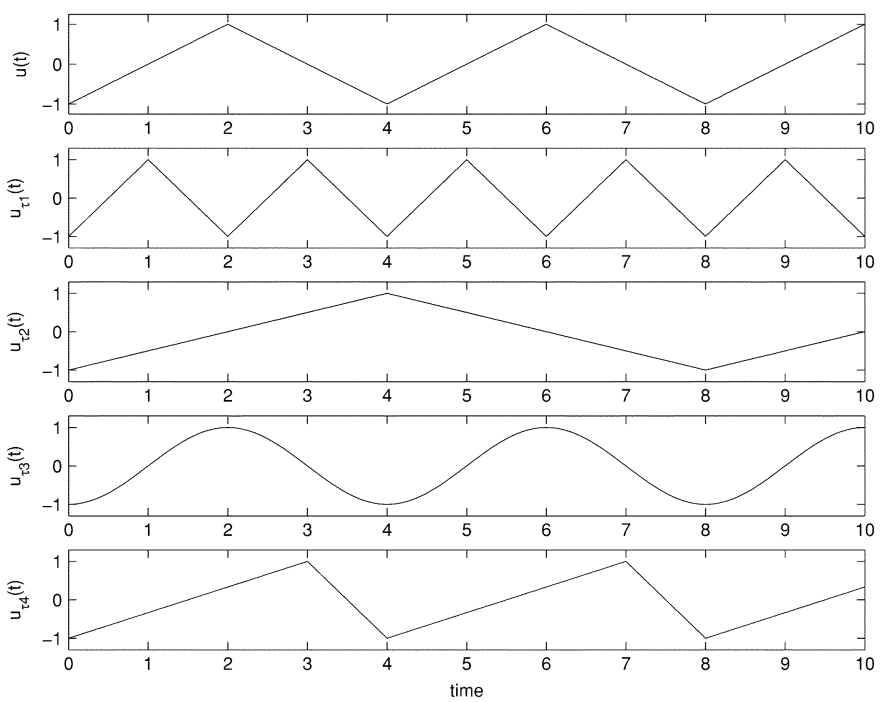

Fig. 7. Time histories of $u, u_{\tau_{1}}, u_{\tau_{2}}, u_{\tau_{3}}$, and $u_{\tau_{4}}$. The inputs $u_{\tau_{1}}$ and $u_{\tau_{2}}$ are identical to $u$ except for their periods, $u_{\tau_{3}}(t)=\sin (\pi t / 2-\pi / 2)$, and $u_{\tau_{4}}$ is a triangle wave with the same period as $u$ but different slope.

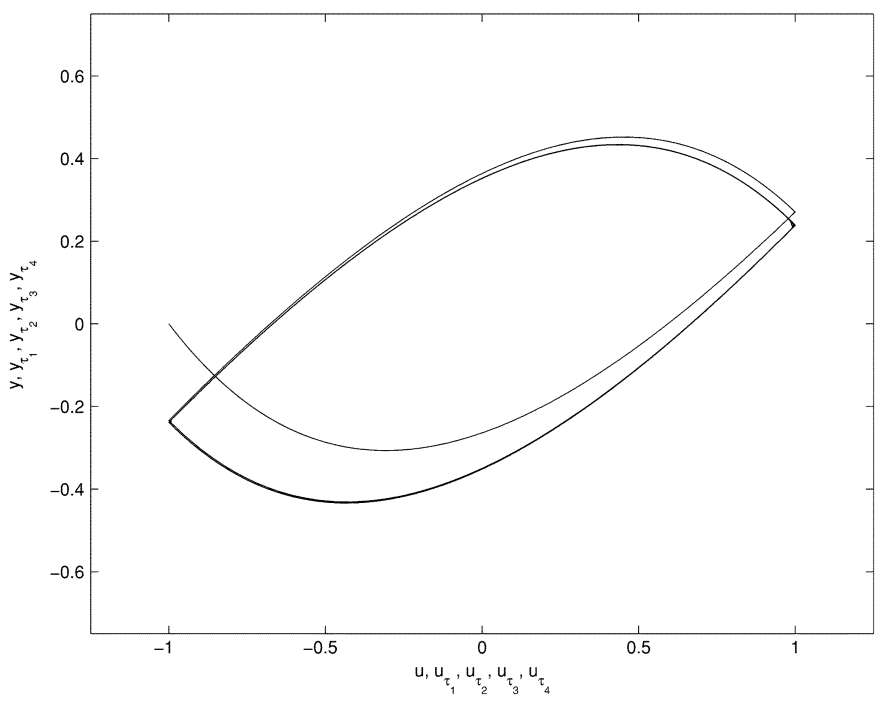

Fig. 8. Input-output maps of $(u, y),\left(u_{\tau_{1}}, y_{\tau_{1}}\right),\left(u_{\tau_{2}}, y_{\tau_{2}}\right),\left(u_{\tau_{3}}, y_{\tau_{3}}\right)$, and $\left(u_{\tau_{4}}, y_{\tau_{4}}\right)$ with $g(\dot{u})=|\dot{u}|$. All of the input-output maps are identical since the model is rate independent.

shows that the input-output maps of (3.10) and (3.11) with different positive time scales are identical. Next, let $g(\dot{u})=\dot{u}^{2}$, which is not positively homogeneous. Fig. 9 shows that the input-output maps of (3.10) and (3.11) depend on the positive time scales.

Example 3.2: Reconsider Example 3.1 with $g(\dot{u})=|\dot{u}|$. Since the model is rate independent, we can reparameterize (3.10) and (3.11) in terms of $u$ to obtain

$$
\begin{aligned}
\frac{\mathrm{d} \hat{x}(u)}{\mathrm{d} u} & = \begin{cases}-\hat{x}(u)+u, & \text { when } u \text { increases } \\
\hat{x}(u)-u, & \text { when } u \text { decreases } \\
0, & \text { otherwise }\end{cases} \\
\hat{y}(u) & =\hat{x}(u), \quad \hat{x}(0)=0 .
\end{aligned}
$$



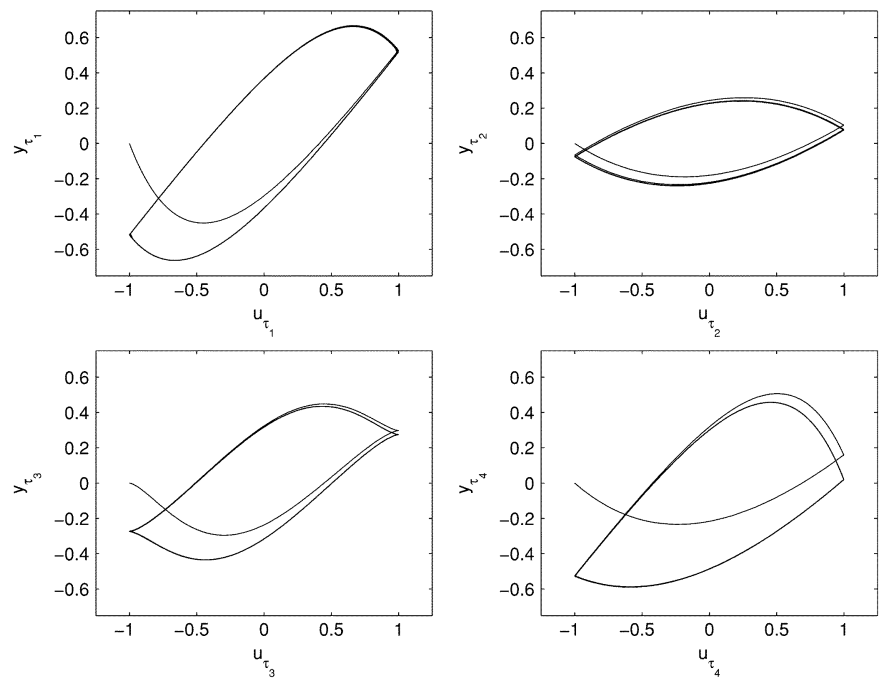

Fig. 9. Input-output maps of $(u, y),\left(u_{\tau_{1}}, y_{\tau_{1}}\right),\left(u_{\tau_{2}}, y_{\tau_{2}}\right),\left(u_{\tau_{3}}, y_{\tau_{3}}\right)$, and $\left(u_{\tau_{4}}, y_{\tau_{4}}\right)$ with $g(\dot{u})=\dot{u}^{2}$. In this case, the input-output map changes with different positive time scales.

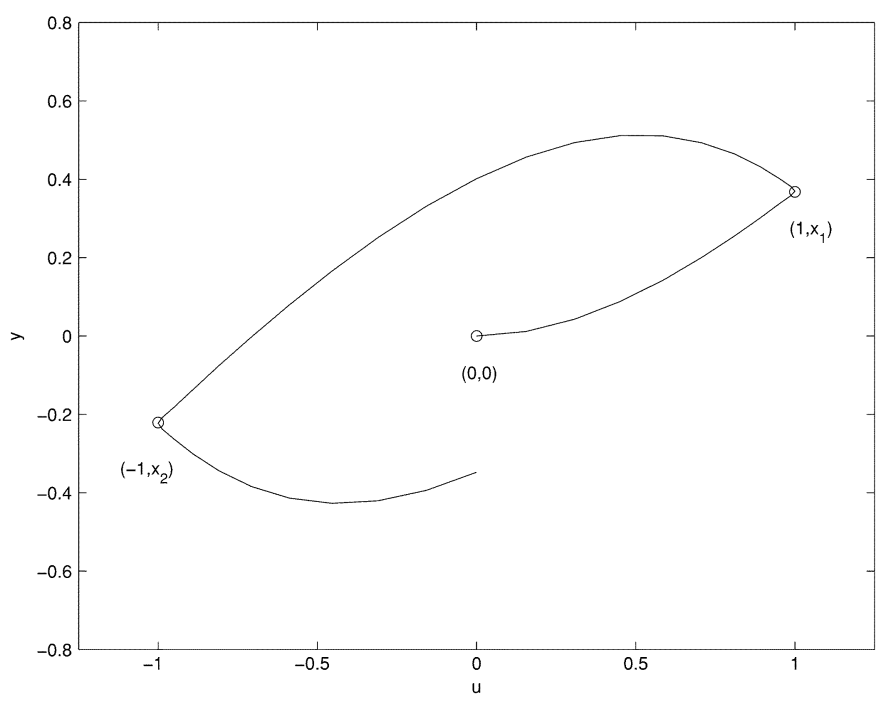

Fig. 10. Input-output map of Example 3.2 with $g(\dot{u})=|\dot{u}|$ and $u(t)=\sin t$.

Consider the piecewise monotonic sinusoidal input $u(t)=$ $\sin t$. When $u$ increases from 0 to $1, \hat{x}(u)$ is given by the forward-time ramp response $\hat{x}(u)$ of

$$
\frac{\mathrm{d} \hat{x}(u)}{\mathrm{d} u}=-\hat{x}(u)+u \quad \hat{x}(0)=0, \quad u \in[0,1]
$$

which yields the branch from $(0,0)$ to $\left(1, x_{1}\right)$ of the input-output map shown in Fig. 10. Now, when $u$ decreases from 1 to $-1, \hat{x}(u)$ is given by the backward-time ramp response $\hat{x}(u)$ of

$$
\frac{\mathrm{d} \hat{x}(u)}{\mathrm{d} u}=\hat{x}(u)-u \quad \hat{x}(1)=x_{1}, \quad u \in[-1,1]
$$

which yields the branch from $\left(1, x_{1}\right)$ to $\left(-1, x_{2}\right)$ of the input-output map shown in Fig. 10.

\section{Rate-IndEPEndent SEMILINEAR DuHem Model}

As a specialization of (3.6) and (3.7), we now consider the rate-independent semilinear Duhem model

$$
\begin{aligned}
\dot{x}(t)= & {\left[\begin{array}{ll}
\dot{u}_{+}(t) I_{n} & \dot{u}_{-}(t) I_{n}
\end{array}\right] } \\
& \times\left(\left[\begin{array}{l}
A_{+} \\
A_{-}
\end{array}\right] x(t)+\left[\begin{array}{l}
B_{+} \\
B_{-}
\end{array}\right] u(t)+\left[\begin{array}{c}
E_{+} \\
E_{-}
\end{array}\right]\right) \\
y(t)= & C x(t)+D u(t), \quad x(0)=x_{0}, \quad t \geq 0
\end{aligned}
$$

where $A_{+} \in \mathbb{R}^{n \times n}, A_{-} \in \mathbb{R}^{n \times n}, B_{+} \in \mathbb{R}^{n}, B_{-} \in \mathbb{R}^{n}, E_{+} \in$ $\mathbb{R}^{n}, E_{-} \in \mathbb{R}^{n}, C \in \mathbb{R}^{1 \times n}$, and $D \in \mathbb{R}$. Note that (4.1) is a rate-independent generalized Duhem model of the form (3.6), (3.7) with $f_{+}(x(t), u(t))=A_{+} x(t)+B_{+} u(t)+$ $E_{+}, f_{-}(x(t), u(t))=A_{-} x(t)+B_{-} u(t)+E_{-}$, and $h(x(t), u(t))=C x(t)+D u(t)$. Reparameterizing (4.1) and (4.2) in terms of $u$ yields

$$
\begin{aligned}
\frac{\mathrm{d} \hat{x}(u)}{\mathrm{d} u} & = \begin{cases}A_{+} \hat{x}(u)+B_{+} u+E_{+}, & \text {when } u \text { increases } \\
A_{-} \hat{x}(u)+B_{-} u+E_{-}, & \text {when } u \text { decreases } \\
0, & \text { otherwise }\end{cases} \\
\hat{y}(u) & =C \hat{x}(u)+D u, \quad \hat{x}\left(u_{0}\right)=x_{0}
\end{aligned}
$$

where $u_{0}=u(0)$. Note that the forward and backward solutions of (4.3) are each given by the sum of a ramp response and a step response. For the following lemma, let $A^{\mathrm{D}}$ denote the Drazin generalized inverse of $A$ and let ind $A$ denote the index of $A$ [24, p. 122].

Lemma 4.1: Let $r_{+} \triangleq$ ind $A_{+}$and $r_{-} \triangleq$ ind $A_{-}$. Then the solution of (4.3) for increasing $u$ is given by

$$
\begin{aligned}
& \hat{x}(u) \\
& \quad=e^{A_{+}\left(u-u_{0}\right)} x_{0}-A_{+}^{\mathrm{D}}\left(u I-u_{0} e^{A_{+}\left(u-u_{0}\right)}\right) B_{+} \\
& -A_{+}^{2 \mathrm{D}}\left(I-e^{A_{+}\left(u-u_{0}\right)}\right) B_{+}-A_{+}^{\mathrm{D}}\left(I-e^{A_{+}\left(u-u_{0}\right)}\right) E_{+} \\
& \quad+\mathcal{X}_{+}\left(u, u_{0}\right)+\mathcal{Y}_{+}\left(u-u_{0}\right), \quad u \geq u_{0}
\end{aligned}
$$

and the solution of (4.3) for decreasing $u$ is given by

$\hat{x}(u)$

$$
\begin{aligned}
= & e^{A_{-}\left(u-u_{0}\right)} x_{0}-A_{-}^{\mathrm{D}}\left(u I-u_{0} e^{A_{-}\left(u-u_{0}\right)}\right) B_{-} \\
& -A_{-}^{2 \mathrm{D}}\left(I-e^{A_{-}\left(u-u_{0}\right)}\right) B_{-}-A_{-}^{\mathrm{D}}\left(I-e^{A_{-}\left(u-u_{0}\right)}\right) E_{-} \\
& +\mathcal{X}_{-}\left(u, u_{0}\right)+\mathcal{Y}_{-}\left(u-u_{0}\right), \quad u \leq u_{0}
\end{aligned}
$$

where

$$
\begin{aligned}
\mathcal{X}_{+}\left(u, u_{0}\right) & \triangleq\left(I-A_{+} A_{+}^{\mathrm{D}}\right) \sum_{k=1}^{r_{+}} \frac{u+k u_{0}}{(k+1) !}\left(u-u_{0}\right)^{k} A_{+}^{k-1} B_{+} \\
\mathcal{X}_{-}\left(u, u_{0}\right) & \triangleq\left(I-A_{-} A_{-}^{\mathrm{D}}\right) \sum_{k=1}^{r_{-}} \frac{u+k u_{0}}{(k+1) !}\left(u-u_{0}\right)^{k} A_{-}^{k-1} B_{-} \\
\mathcal{Y}_{+}(u) & \triangleq\left(I-A_{+} A_{+}^{\mathrm{D}}\right) \sum_{k=1}^{r_{+}} \frac{1}{k !} u^{k} A_{+}^{k-1} E_{+} \\
\mathcal{Y}_{-}(u) & \triangleq\left(I-A_{-} A_{-}^{\mathrm{D}}\right) \sum_{k=1}^{r_{-}} \frac{1}{k !} u^{k} A_{-}^{k-1} E_{-} .
\end{aligned}
$$



$\mathbb{R}^{n \times n}$

Proof: First, we recall from [24, p. 176] that, for $A \in$

$$
\int_{0}^{u} e^{A v} \mathrm{~d} v=A^{\mathrm{D}}\left(e^{A u}-I\right)+\left(I-A A^{\mathrm{D}}\right) \sum_{k=1}^{r} \frac{1}{k !} u^{k} A^{k-1}
$$

where $r \triangleq \operatorname{ind} A$. Now, suppose $u$ is increasing. Then, with the initial condition $\hat{x}(u)=u_{0}$, the solution $\hat{x}(u)$ of (4.3) is given by

$$
\begin{aligned}
\hat{x}(u) & \\
= & e^{A_{+}\left(u-u_{0}\right)} x_{0}+\int_{u_{0}}^{u} e^{A_{+}(u-v)}\left(B_{+} v+E_{+}\right) \mathrm{d} v \\
= & e^{A_{+}\left(u-u_{0}\right)} x_{0}-A_{+}^{\mathrm{D}}\left(u I-u_{0} e^{A_{+}\left(u-u_{0}\right)}\right) B_{+} \\
& -A_{+}^{2 \mathrm{D}}\left(I-e^{A_{+}\left(u-u_{0}\right)}\right) B_{+}-A_{+}^{\mathrm{D}}\left(I-e^{A_{+}\left(u-u_{0}\right)}\right) E_{+} \\
& +\mathcal{X}_{+}\left(u, u_{0}\right)+\mathcal{Y}_{+}\left(u-u_{0}\right)
\end{aligned}
$$

which proves (4.5). Now suppose $u$ is decreasing and let $\hat{x}_{-}(u) \triangleq \hat{x}(-u)$. Then, the solution $\hat{x}(u)$ of (4.3) with $u$ decreasing from $u_{0}$ is equivalent to $\hat{x}_{-}(u)$ with $u$ increasing from $-u_{0}$. For all $u \geq-u_{0}$, it follows from (4.3) that

$$
\frac{\mathrm{d} \hat{x}(-u)}{\mathrm{d}(-u)}=A_{-} \hat{x}(-u)+B_{-}(-u)+E_{-}
$$

that is

$$
-\frac{\mathrm{d} \hat{x}_{-}(u)}{\mathrm{d} u}=A_{-} \hat{x}_{-}(u)-B_{-} u+E_{-} .
$$

Therefore

$$
\frac{\mathrm{d} \hat{x}_{-}(u)}{\mathrm{d} u}=-A_{-} \hat{x}_{-}(u)+B_{-} u-E_{-} .
$$

Hence, with the initial condition $\hat{x}_{-}\left(-u_{0}\right)=x_{0}, \hat{x}_{-}(u)$ is given by

$$
\begin{aligned}
\hat{x}_{-}(u)= & e^{-A_{-}\left(u+u_{0}\right)} x_{0}+\int_{-u_{0}}^{u} e^{-A_{-}(u-v)}\left(B_{-} v-E_{+}\right) \mathrm{d} v \\
= & e^{-A_{-}\left(u+u_{0}\right)} x_{0}+A_{-}^{\mathrm{D}}\left(u I+u_{0} e^{-A_{-}\left(u+u_{0}\right)}\right) B_{-} \\
& -A_{-}^{2 \mathrm{D}}\left(I-e^{-A_{+}\left(u+u_{0}\right)}\right) B_{-}+\left(I-A_{-} A_{-}^{\mathrm{D}}\right) \\
& \times \sum_{k=1}^{r_{-}} \frac{u-k u_{0}}{(k+1) !}\left(u+u_{0}\right)^{k}\left(-A_{-}\right)^{k-1} B_{-} \\
& -A_{-}^{\mathrm{D}}\left(I-e^{-A_{-}\left(u+u_{0}\right)}\right) E_{-}\left(I-A_{-} A_{-}^{\mathrm{D}}\right) \\
& \times \sum_{k=1}^{r_{-}} \frac{1}{k !}\left(u+u_{0}\right)^{k}\left(-A_{-}\right)^{k-1} E_{-} .
\end{aligned}
$$

Since $\hat{x}(u)=\hat{x}_{-}(-u)$, we have, for $u \leq u_{0}$

$\hat{x}(u)$

$$
\begin{aligned}
= & e^{A_{-}\left(u-u_{0}\right)} x_{0}-A_{-}^{\mathrm{D}}\left(u I-u_{0} e^{A_{-}\left(u-u_{0}\right)}\right) B_{-} \\
& -A_{-}^{2 \mathrm{D}}\left(I-e^{A_{-}\left(u-u_{0}\right)}\right) B_{-}-A_{-}^{\mathrm{D}}\left(I-e^{A_{-}\left(u-u_{0}\right)}\right) E_{-} \\
& +\mathcal{X}_{-}\left(u, u_{0}\right)+\mathcal{Y}_{-}\left(u-u_{0}\right) .
\end{aligned}
$$

Remark 4.1: If $r_{+} \leq 1$, then $A_{+}^{\mathrm{D}}=A_{+}^{\#}$ and thus $\mathcal{X}_{+}\left(u, u_{0}\right)=(1 / 2)\left(u^{2}-u_{0}^{2}\right)\left(I-A_{+} A_{+}^{\#}\right) B_{+}$and $\mathcal{Y}_{+}(u)=\left(u-u_{0}\right)\left(I-A_{+} A_{+}^{\#}\right) E_{+}$, where $A_{+}^{\#}$ is the group generalized inverse of $A_{+}$[24, p. 124]. If $r_{+}=0$, that is, $A_{+}$is nonsingular, then $A_{+}^{\mathrm{D}}=A_{+}^{-1}$ and, thus, $\mathcal{X}_{+}\left(u, u_{0}\right)=\mathcal{Y}_{+}(u)=0$. Similarly, if $r_{-}=1, A_{-}^{\mathrm{D}}=A_{-}^{\#}$ and thus $\mathcal{X}_{-}\left(u, u_{0}\right)=(1 / 2)\left(u^{2}-u_{0}^{2}\right)\left(I-A_{-} A_{-}^{\#}\right)$ and $\mathcal{Y}_{-}(u)=\left(u-u_{0}\right)\left(I-A_{-} A_{-}^{\#}\right) E_{-}$. Furthermore, if $r_{-}=0$, then $A_{-}^{\mathrm{D}}=A_{-}^{-1}$ and $\mathcal{X}_{-}\left(u, u_{0}\right)=\mathcal{Y}_{-}(u)=0$.

For the following result, let the limiting input-output map $\mathcal{F}_{\infty}(u, y)$ be the set of points $z \in \mathbb{R}^{2}$ such that there exists a divergent sequence $\left\{t_{i}\right\}$ in $[0, \infty)$ satisfying $\lim _{i \rightarrow \infty}\left\|\left(u\left(t_{i}\right), y\left(t_{i}\right)\right)-z\right\|=0$. Furthermore, let $\rho(A)$ denote the spectral radius of $A \in \mathbb{R}^{n \times n}$. We now state the main result on the existence of the limiting periodic input-output map of a rate-independent semilinear Duhem model.

Theorem 4.1: Consider the rate-independent semilinear Duhem model (4.1), (4.2), where $u:[0, \infty) \rightarrow\left[u_{\min }, u_{\max }\right]$ is continuous, piecewise $C^{1}$, and periodic with period $\alpha$ and has exactly one local maximum $u_{\max }$ in $[0, \alpha)$ and exactly one local minimum $u_{\min }$ in $[0, \alpha)$. Furthermore, define $\beta \triangleq u_{\max }-u_{\min }$ and assume that $\rho\left(e^{\beta A_{+}} e^{-\beta A_{-}}\right)<1$. Then, for all $T>0$, (4.1) has a unique periodic solution $x_{T}:[0, \infty) \rightarrow \mathbb{R}^{n}$, the limiting periodic input-output map $\mathcal{H}_{\infty}(u)$ exists, and the limiting input-output map $\mathcal{F}_{\infty}(u, y)$ is given by $\mathcal{F}_{\infty}(u, y)=\mathcal{H}_{\infty}(u)$. Specifically

$$
\mathcal{H}_{\infty}(u)=\left\{\left(u, \hat{y}_{+}(u)\right)\right\} \cup\left\{\left(u, \hat{y}_{-}(u)\right)\right\}
$$

where

$$
\hat{y}_{+}(u) \triangleq C \hat{x}_{+}(u)+D u \quad \hat{y}_{-}(u) \triangleq C \hat{x}_{-}(u)+D u
$$

and

$$
\begin{aligned}
& \hat{x}_{+}(u) \triangleq e^{A_{+}\left(u-u_{\min }\right)} \hat{x}_{+}-A_{+}^{\mathrm{D}}\left[u I-u_{\min } e^{A_{+}\left(u-u_{\min }\right)}\right] B_{+} \\
& -A_{+}^{2 \mathrm{D}}\left[I-e^{A_{+}\left(u-u_{\min }\right)}\right] B_{+} \\
& -A_{+}^{\mathrm{D}}\left(I-e^{A_{+}\left(u-u_{\min }\right)}\right) E_{+}+\mathcal{X}_{+}\left(u, u_{\min }\right) \\
& +\mathcal{Y}_{+}\left(u-u_{\min }\right) \\
& \hat{x}_{-}(u) \triangleq e^{A_{-}\left(u-u_{\max }\right)} \hat{x}_{-}-A_{-}^{\mathrm{D}}\left[u I-u_{\max } e^{A_{-}\left(u-u_{\max }\right)}\right] B_{-} \\
& -A_{-}^{2 \mathrm{D}}\left[I-e^{A_{-}\left(u-u_{\max }\right)}\right] B_{-} \\
& -A_{-}^{\mathrm{D}}\left(I-e^{A_{-}\left(u-u_{\max }\right)}\right) E_{-} \\
& +\mathcal{X}_{-}\left(u, u_{\max }\right)+\mathcal{Y}_{-}\left(u-u_{\max }\right) \\
& \hat{x}_{+} \triangleq\left(I-e^{-\beta A_{-}} e^{\beta A_{+}}\right)^{-1}\left(e^{-\beta A_{-}} \mathcal{Z}_{+}+\mathcal{Z}_{-}\right) \\
& \hat{x}_{-} \triangleq\left(I-e^{\beta A_{+}} e^{-\beta A_{-}}\right)^{-1}\left(e^{\beta A_{+}} \mathcal{Z}_{-}+\mathcal{Z}_{+}\right) \\
& \mathcal{Z}_{+} \triangleq-A_{+}^{\mathrm{D}}\left(u_{\text {max }} I-u_{\min } e^{\beta A_{+}}\right) B_{+} \\
& -A_{+}^{2 \mathrm{D}}\left(I-e^{\beta A_{+}}\right) B_{+}-A_{+}^{\mathrm{D}}\left(I-e^{\beta A_{+}}\right) E_{+} \\
& +\mathcal{X}_{+}\left(u_{\max }, u_{\min }\right)+\mathcal{Y}_{+}(\beta) \\
& \mathcal{Z}_{-} \triangleq-A_{-}^{\mathrm{D}}\left(u_{\min } I-u_{\max } e^{-\beta A_{-}}\right) B_{-} \\
& -A_{-}^{2 \mathrm{D}}\left(I-e^{-\beta A_{-}}\right) B_{-}-A_{-}^{\mathrm{D}}\left(I-e^{-\beta A_{-}}\right) E_{-} \\
& +\mathcal{X}_{-}\left(u_{\min }, u_{\max }\right)+\mathcal{Y}_{-}(-\beta) \text {. }
\end{aligned}
$$


Proof: Let $T>0$, define $u_{T}(t)=u(\alpha t / T), t \geq 0$, and define

$$
x_{T}(t) \triangleq\left\{\begin{array}{ll}
\hat{x}_{+}\left(u_{T}(t)\right), & \text { when } u_{T}(t) \text { increases, } \\
\hat{x}_{-}\left(u_{T}(t)\right), & \text { when } u_{T}(t) \text { decreases, }
\end{array} \quad t \geq 0 .\right.
$$

Since

$$
\begin{aligned}
\hat{x}_{+} & \left(u_{\max }\right) \\
= & e^{\beta A_{+}}\left(I-e^{-\beta A_{-}} e^{\beta A_{+}}\right)^{-1}\left(e^{-\beta A_{-}} \mathcal{Z}_{+}+\mathcal{Z}_{-}\right)+\mathcal{Z}_{+} \\
= & \left(I-e^{\beta A_{+}} e^{-\beta A_{-}}\right)^{-1} e^{\beta A_{+}}\left(e^{-\beta A_{-}} \mathcal{Z}_{+}+\mathcal{Z}_{-}\right)+\mathcal{Z}_{+} \\
= & \left(I-e^{\beta A_{+}} e^{-\beta A_{-}}\right)^{-1}\left[e^{\beta A_{+}} e^{-\beta A_{-}} \mathcal{Z}_{+}+e^{\beta A_{+}} \mathcal{Z}_{-}\right. \\
& \left.+\left(I-e^{\beta A_{+}} e^{-\beta A_{-}}\right) \mathcal{Z}_{+}\right] \\
= & \left(I-e^{\beta A_{+}} e^{-\beta A_{-}}\right)^{-1}\left(e^{\beta A_{+}} \mathcal{Z}_{-}+\mathcal{Z}_{+}\right) \\
= & \hat{x}_{-}\left(u_{\max }\right)
\end{aligned}
$$

and

$$
\begin{aligned}
\hat{x}_{-} & \left(u_{\min }\right) \\
= & e^{-\beta A_{-}}\left(I-e^{\beta A_{+}} e^{-\beta A_{-}}\right)^{-1}\left(e^{\beta A_{+}} \mathcal{Z}_{-}+\mathcal{Z}_{+}\right)+\mathcal{Z}_{-} \\
= & \left(I-e^{-\beta A_{-}} e^{\beta A_{+}}\right)^{-1} e^{-\beta A_{-}}\left(e^{\beta A_{+}} \mathcal{Z}_{-}+\mathcal{Z}_{+}\right)+\mathcal{Z}_{-} \\
= & \left(I-e^{-\beta A_{-}} e^{\beta A_{+}}\right)^{-1}\left[e^{-\beta A_{-}} e^{\beta A_{+}} \mathcal{Z}_{-}+e^{-\beta A_{-}} \mathcal{Z}_{+}\right. \\
& \left.+\left(I-e^{-\beta A_{-}} e^{\beta A_{+}}\right) \mathcal{Z}_{-}\right] \\
= & \left(I-e^{-\beta A_{-}} e^{\beta A_{+}}\right)^{-1}\left(e^{-\beta A_{-}} \mathcal{Z}_{+}+\mathcal{Z}_{-}\right) \\
= & \hat{x}_{+}\left(u_{\min }\right)
\end{aligned}
$$

and since $u_{T}$ continuous, piecewise $C^{1}$, and periodic with pe$\operatorname{riod} T$, it follows that $x_{T}$ is continuous, piecewise $C^{1}$, and periodic with period $T$. Furthermore, Lemma 4.1 implies that $\hat{x}_{+}(u)$ is the solution of (4.3) with $u=u_{\min }$ and $x_{0}=\hat{x}_{+}$for increasing $u$ and $\hat{x}_{-}(u)$ is the solution of (4.3) with $u=u_{\max }$ and $x_{0}=\hat{x}_{-}$for decreasing $u$. Hence $x_{T}$ is a periodic solution of (4.1), (4.2) with $u=u_{T}$ and, thus, the periodic input-output map is given by $\mathcal{H}_{T}\left(u_{T}, y_{T}\right) \triangleq\left\{\left(u_{T}(t), C x_{T}(t)+D u_{T}(t)\right)\right.$ : $t \in[0, \infty)\}=\mathcal{H}_{\infty}\left(u_{T}\right)$. Since (4.1), (4.2) is rate independent, it follows that $\mathcal{H}_{T}\left(u_{T}, y_{T}\right)=\mathcal{H}_{\infty}(u)$, for all $T>0$.

Now, to prove that $x_{T}$ is the unique periodic solution of (4.1), (4.2), let $\tilde{x}_{T}(t), t \geq 0$ satisfy (4.1) with $u=u_{T}$. Let $t_{\max } \triangleq$ $\min \left\{t \geq 0: u_{T}(t)=u_{\max }\right\}$ and $t_{\min } \triangleq \min \left\{t \geq 0: u_{T}(t)=\right.$ $\left.u_{\min }\right\}$, and for $k=0,1,2, \ldots$, define $\hat{x}_{k_{+}} \triangleq \tilde{x}_{T}\left(t_{\min }+k T\right)$ and $\hat{x}_{k_{-}} \triangleq \tilde{x}_{T}\left(t_{\max }+k T\right)$. Without loss of generality, let $t_{\max }<t_{\min }$. Analogous arguments hold for the case $t_{\max }>$ $t_{\min }$. Since $u_{T}(t)$ is periodic and has exactly one local maximum and exactly one local minimum in each period, Lemma 4.1 implies that $\hat{x}_{0_{-}}$is given by (4.5) with $u=u_{\max }$ as

$$
\begin{aligned}
\hat{x}_{0_{-}}= & e^{A_{+}\left(u_{\max }-u_{0}\right)} x_{0} \\
& -A_{+}^{\mathrm{D}}\left(u_{\max } I-u_{0} e^{A_{+}\left(u_{\max }-u_{0}\right)}\right) B_{+} \\
& -A_{+}^{2 \mathrm{D}}\left(I-e^{A_{+}\left(u_{\max }-u_{0}\right)}\right) B_{+} \\
& -A_{+}^{\mathrm{D}}\left(I-e^{A_{+}\left(u_{\max }-u_{0}\right)}\right) E_{+} \\
& +\mathcal{X}_{+}\left(u_{\max }, u_{0}\right)+\mathcal{Y}_{+}\left(u_{\max }-u_{0}\right)
\end{aligned}
$$

and, for $k=1,2,3, \ldots, \hat{x}_{k_{-}}$is given by (4.5) with $u=u_{\max }, u_{0}=u_{\min }$, and $x_{0}=\hat{x}_{(k-1)_{+}}$as

$$
\hat{x}_{k_{-}}=e^{\beta A_{+}} \hat{x}_{(k-1)_{+}}+\mathcal{Z}_{+} \text {. }
$$

Similarly, for $k=1,2,3, \ldots, \hat{x}_{k_{+}}$is given by (4.6) with $u=$ $u_{\min }, u_{0}=u_{\max }$, and $x_{0}=\hat{x}_{k_{-}}$as

$$
\hat{x}_{k_{+}}=e^{-\beta A_{-}} \hat{x}_{k_{-}}+\mathcal{Z}_{-} .
$$

Since $\hat{x}_{k_{+}}$is obtained by a sequence of forward and backward solutions of (4.3) for $k=1,2,3, \ldots, \hat{x}_{k_{+}}$is given by the affine map

$$
\begin{aligned}
\hat{x}_{k_{+}} & =e^{-\beta A_{-}}\left(e^{\beta A_{+}} \hat{x}_{(k-1)_{+}}+\mathcal{Z}_{+}\right)+\mathcal{Z}_{-} \\
& =\left(e^{-\beta A_{-}} e^{\beta A_{+}}\right) \hat{x}_{(k-1)_{+}}+e^{-\beta A_{-}} \mathcal{Z}_{+}+\mathcal{Z}_{-} .
\end{aligned}
$$

Similarly, for $k=1,2,3, \ldots$, since $\hat{x}_{k_{-}}$is also obtained by a sequence of backward and forward solutions of (4.3), $\hat{x}_{k_{-}}$is given by the affine map

$$
\begin{aligned}
\hat{x}_{k_{-}} & =e^{\beta A_{+}}\left(e^{-\beta A_{-}} \hat{x}_{(k-1)_{-}}+\mathcal{Z}_{-}\right)+\mathcal{Z}_{+} \\
& =\left(e^{\beta A_{+}} e^{-\beta A_{-}}\right) \hat{x}_{(k-1)_{-}}+e^{\beta A_{+}} \mathcal{Z}_{-}+\mathcal{Z}_{+} .
\end{aligned}
$$

Since $\rho\left(e^{\beta A_{+}} e^{-\beta A_{-}}\right)<1$ and hence $\rho\left(e^{-\beta A_{-}} e^{\beta A_{+}}\right)<1$, it follows that

$$
\begin{aligned}
& \lim _{k \rightarrow \infty} \hat{x}_{k_{+}}=\left(I-e^{-\beta A_{-}} e^{\beta A_{+}}\right)^{-1}\left(e^{-\beta A_{-}} \mathcal{Z}_{+}+\mathcal{Z}_{-}\right)=\hat{x}_{+} \\
& \text {and } \\
& \lim _{k \rightarrow \infty} \hat{x}_{k_{-}}=\left(I-e^{\beta A_{+}} e^{-\beta A_{-}}\right)^{-1}\left(e^{\beta A_{+}} \mathcal{Z}_{-}+\mathcal{Z}_{+}\right)=\hat{x}_{-} .
\end{aligned}
$$

Therefore, Lemma 4.1 implies that, as $t \rightarrow \infty$ and, thus, $k \rightarrow$ $\infty, \tilde{x}_{T}(t)$ converges to the forward solution of (4.3) with $u=$ $u_{\max }, u_{0}=u_{\min }$, and $x_{0}=\hat{x}_{+}$, and the backward solution of (4.3) with $u=u_{\min }, u_{0}=u_{\max }$, and $x_{0}=\hat{x}_{-}$, which is $x_{T}(t)$. Therefore, $x_{T}$ is the unique periodic solution of (4.1) and (4.2) with $u=u_{T}$.

Finally, to prove $\mathcal{F}_{\infty}(u, y)=\mathcal{H}_{\infty}(u)$, let $\left(z_{u}, z_{y}\right) \in \mathcal{H}_{\infty}(u)$ and let $z_{y}=y_{+}\left(z_{u}\right)$ without loss of generality. Analogous arguments hold for the case $z_{y}=y_{-}\left(z_{u}\right)$. Let $t_{z} \triangleq \min \{t \geq$ $\left.0: u_{T}(t)=z_{u}\right\}$, and define $\left\{t_{k}\right\} \triangleq\left\{t_{\text {min }}+t_{z}+k T\right\}$. Then, $\lim _{k \rightarrow \infty}\left\|\left(u_{T}\left(t_{k}\right), C \tilde{x}_{T}\left(t_{k}\right)+D u_{T}\left(t_{k}\right)\right)-\left(z_{u}, z_{y}\right)\right\|=0$, and thus $\mathcal{F}_{\infty}\left(u_{T}, y_{T}\right)=\mathcal{H}_{\infty}(u)$. Since the model is rate independent, $\mathcal{F}_{\infty}\left(u_{T_{1}}, y_{T_{1}}\right)=\mathcal{F}_{\infty}\left(u_{T_{2}}, y_{T_{2}}\right)=\mathcal{H}_{\infty}(u)$ for all $T_{1}, T_{2}>0$, as required.

As a special case of (4.1), (4.2), consider the rate-independent semilinear Duhem model

$\dot{x}(t)=\left[\begin{array}{ll}\dot{u}_{+}(t) I_{n} & \dot{u}_{-}(t) I_{n}\end{array}\right]\left(\left[\begin{array}{l}h_{+} A \\ h_{-} A\end{array}\right] x(t)+\left[\begin{array}{l}h_{+} B \\ h_{-} B\end{array}\right] u(t)\right)$

$$
y(t)=C x(t), \quad x(0)=x_{0}, \quad t \geq 0
$$

where $A \in \mathbb{R}^{n \times n}, B \in \mathbb{R}^{n}, h_{+}, h_{-} \in \mathbb{R}$, and $C \in \mathbb{R}^{1 \times n}$. Then, (4.15) and (4.16) can be rewritten as

$$
\begin{aligned}
& \dot{x}(t)=(A x(t)+B u(t)) g(\dot{u}(t)) \\
& y(t)=C x(t), \quad x(0)=x_{0}, \quad t \geq 0
\end{aligned}
$$

where $g$ is given by (3.1). The following result is the specialization of Theorem 4.1 to (4.15) and (4.16).

Corollary 4.1: Consider the rate-independent semilinear Duhem model (4.15), (4.16), where $u:[0, \infty) \rightarrow\left[u_{\min }, u_{\max }\right]$ is continuous, piecewise $C^{1}$, and periodic with period $\alpha$ and 


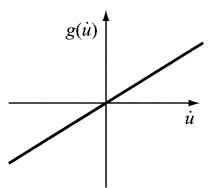

(a)

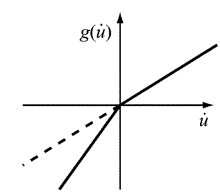

(b)

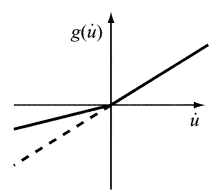

(c)

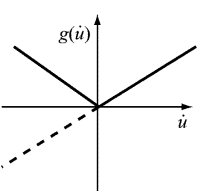

(d)
Fig. 11. Illustration of positively homogeneous functions $g$ for asymptotically stable $A$. (a) Not hysteretic. (b) May not converge to $\mathcal{H}_{\infty}$ since $h_{-}>h_{+}$. (c), (d) Will converge to $\mathcal{H}_{\infty}$.

has exactly one local maximum $u_{\max }$ in $[0, \alpha)$ and exactly one local minimum $u_{\min }$ in $[0, \alpha)$. Furthermore, suppose that $A$ is asymptotically stable and $h_{-}<h_{+}$. Then, for all $T>0$, (4.15) has a unique periodic solution $x_{T}:[0, \infty) \rightarrow \mathbb{R}^{n}$, the limiting periodic input-output map $\mathcal{H}_{\infty}(u)$ exists, and the limiting input-output map $\mathcal{F}_{\infty}(u, y)$ of (4.1) and (4.2) is given by $\mathcal{F}_{\infty}(u, y)=\mathcal{H}_{\infty}(u)$. Specifically

$$
\mathcal{H}_{\infty}(u)=\left\{\left(u, \hat{y}_{+}(u)\right)\right\} \cup\left\{\left(u, \hat{y}_{-}(u)\right)\right\}
$$

where

$$
\begin{aligned}
\hat{y}_{+}(u)= & C e^{h_{+} A\left(u-u_{\min }\right)} \hat{x}_{+}-C A^{-1} \\
& \times\left[u I-u_{\min } e^{h_{+} A\left(u-u_{\min }\right)}\right] B \\
& -C h_{+}^{-1} A^{-2}\left[I-e^{h_{+} A\left(u-u_{\min }\right)}\right] B \\
& -C A^{-1}\left(I-e^{h_{+} A\left(u-u_{\min }\right)}\right) E+D u \\
\hat{y}_{-}(u)= & C e^{h_{-} A\left(u-u_{\max }\right)} \hat{x}_{-}-C A^{-1} \\
& \times\left[u I-u_{\max } e^{h_{-} A\left(u-u_{\max }\right)}\right] B \\
& -C h_{-}^{-1} A^{-2}\left[I-e^{h_{-} A\left(u-u_{\max }\right)}\right] B \\
& -C A^{-1}\left(I-e^{h_{-} A\left(u-u_{\max }\right)}\right) E+D u
\end{aligned}
$$

and

$$
\begin{aligned}
\hat{x}_{+} \triangleq & \left(I-e^{\beta\left(h_{+}-h_{-}\right) A}\right)^{-1}\left(e^{-\beta h_{-} A} \mathcal{Z}_{+}+\mathcal{Z}_{-}\right) \\
\hat{x}_{-} \triangleq & \left(I-e^{\beta\left(h_{+}-h_{-}\right) A}\right)^{-1}\left(e^{\beta h_{+} A} \mathcal{Z}_{-}+\mathcal{Z}_{+}\right) \\
\mathcal{Z}_{+} \triangleq & -A^{-1}\left(u_{\max } I-u_{\min } e^{\beta h_{+} A}\right) B \\
& -h_{+}^{-1} A^{-2}\left(I-e^{\beta h_{+} A}\right) B-A^{-1}\left(I-e^{\beta h_{+} A}\right) E \\
\mathcal{Z}_{-} \triangleq & -A^{-1}\left(u_{\min } I-u_{\max } e^{-\beta h_{-} A}\right) B \\
& -h_{-}^{-1} A^{-2}\left(I-e^{-\beta h_{-} A}\right) B-A^{-1}\left(I-e^{-\beta h_{-}}\right) E .
\end{aligned}
$$

Proof: First note that

$$
e^{\beta h_{+} A} e^{-\beta h_{-} A}=e^{-\beta h_{-} A} e^{\beta h_{+} A}=e^{\beta\left(h_{+}-h_{-}\right) A} .
$$

Since $h_{-}<h_{+}$and $A$ is asymptotically stable, it follows that $\rho\left(e^{\beta\left(h_{+}-h_{-}\right) A}\right)<1$. The result is now a consequence of Theorem 4.1.

Consider the rate-independent semilinear Duhem model (4.17), (4.18) and assume that $A$ is asymptotically stable. Then Corollary 4.1 implies that the convergence of the input-output map to the limiting input-output map depends on the leftand right-hand slopes of $g$ at the origin. Fig. 11 illustrates several positively homogeneous functions $g$. If $h_{+}=h_{-}$as in Fig. 11(a), then the positive orbit and the negative orbit of the reparameterized model are identical and thus the model is not hysteretic. If $h_{-}<h_{+}$, it follows from Corollary 4.1 that for the functions $g$ shown in Fig. 11(c) and (d) the rate-independent semilinear Duhem model has a limiting periodic input-output map.

Example 4.1: The ferromagnetic material model (1.2) can be rewritten as

$$
\begin{aligned}
\dot{B}(t)=\left[\dot{H}_{+}\right. & \left.(t) \quad \dot{H}_{-}(t)\right] \\
& \times\left(\left[\begin{array}{c}
-a \\
a
\end{array}\right] B(t)+\left[\begin{array}{c}
a b \\
-a b
\end{array}\right] H(t)+\left[\begin{array}{l}
c \\
c
\end{array}\right]\right)
\end{aligned}
$$

where $\dot{H}_{+}(t) \triangleq \max \{0, \dot{H}(t)\}$ and $\dot{H}_{-}(t) \triangleq \min \{0, \dot{H}(t)\}$. Note that (4.26) is a rate-independent semilinear Duhem model of the form (4.1). Consider the parameters from [2]

$$
a=0.02125 \quad b=0.100 \quad c=0.04361
$$

and let $H(t)=30 \sin t$. Since $\rho\left(e^{-60 a} e^{-60 a}\right)=0.0781<1$, it follows from Theorem 4.1 that the limiting periodic input-output map exists and $(H(t), B(t))$ converges to the limiting input-output map as $t \rightarrow \infty$, as shown in Fig. 3 .

Example 4.2: Consider the rate-independent semilinear Duhem model (4.15) and (4.16) with

$$
\begin{aligned}
A & =\left[\begin{array}{cc}
-1 & 4 \\
-4 & -1
\end{array}\right] \quad B=\left[\begin{array}{l}
0 \\
1
\end{array}\right] \quad C=\left[\begin{array}{ll}
0 & 1
\end{array}\right] \\
x_{0} & =\left[\begin{array}{ll}
0.15 & 0.15
\end{array}\right]^{\mathrm{T}} .
\end{aligned}
$$

Suppose $u(t)=\sin t, t \geq 0$ and let $h_{+}=1$ and $h_{-}=-1$. Then since $A$ is asymptotically stable and $h_{-}<h_{+}$, Corollary 4.1 implies that the limiting periodic input-output map exists and the input-output map converges to the limiting input-output map as $t \rightarrow \infty$, as shown in Fig. 12(a). Now, let $h_{+}=1$ and $h_{-}=1.1$. Then $h_{-}>h_{+}$and the input-output map of $u(t)$ and $y(t)$ does not converge, as shown in Fig. 12(b). In this case, $\rho\left(e^{2 h_{+} A} e^{-2 h_{-} A}\right)=1.2214>1$.

\section{RATE-DEPENDENT SEMILINEAR DUHEM MODEL}

As an alternative specialization of (2.1) and (2.2), we consider the rate-dependent semilinear Duhem model

$$
\begin{aligned}
\dot{x}(t)= & {\left[\begin{array}{ll}
g_{+}(\dot{u}(t)) I_{n} & g_{-}(\dot{u}(t)) I_{n}
\end{array}\right] } \\
& \times\left(\left[\begin{array}{l}
A_{+} \\
A_{-}
\end{array}\right] x(t)+\left[\begin{array}{l}
B_{+} \\
B_{-}
\end{array}\right] u(t)+\left[\begin{array}{l}
E_{+} \\
E_{-}
\end{array}\right]\right) \\
y(t)= & C x(t)+D u(t) \quad x(0)=x_{0}, \quad t \geq 0
\end{aligned}
$$

where $A_{+} \in \mathbb{R}^{n \times n}, A_{-} \in \mathbb{R}^{n \times n}, B_{+} \in \mathbb{R}^{n}, B_{-} \in \mathbb{R}^{n}, E_{+} \in$ $\mathbb{R}^{n}, E_{-} \in \mathbb{R}^{n}, C \in \mathbb{R}^{1 \times n}$, and $D \in \mathbb{R}$, and where $g_{+}: \mathbb{R} \rightarrow$ $\mathbb{R}$ and $g_{-}: \mathbb{R} \rightarrow \mathbb{R}$ are continuous and satisfy $g_{+}(u)=0$ for $u \leq 0$, and $g_{-}(u)=0$ for $u \geq 0$. Note that (5.1), (5.2) is a rate-independent semilinear Duhem model (4.1), (4.2) if $g_{+}(\dot{u}(t))=\dot{u}_{+}(t)$ and $g_{-}(\dot{u}(t))=\dot{u}_{-}(t)$. Furthermore, note that $(5.1),(5.2)$ is a generalized Duhem model of the form (2.1), (2.2) with $f(x(t), u(t))=\left[A_{+} x(t)+B_{+} u(t)+E_{+} A_{-} x(t)+\right.$ $\left.B_{-} u(t)+E_{-}\right]$and $g(\dot{u}(t))=\left[g_{+}(\dot{u}(t)) I_{n} g_{-}(\dot{u}(t)) I_{n}\right]^{\mathrm{T}}$. Since $g$ is not necessarily positively homogeneous, (5.1) and (5.2) are generally rate dependent. 


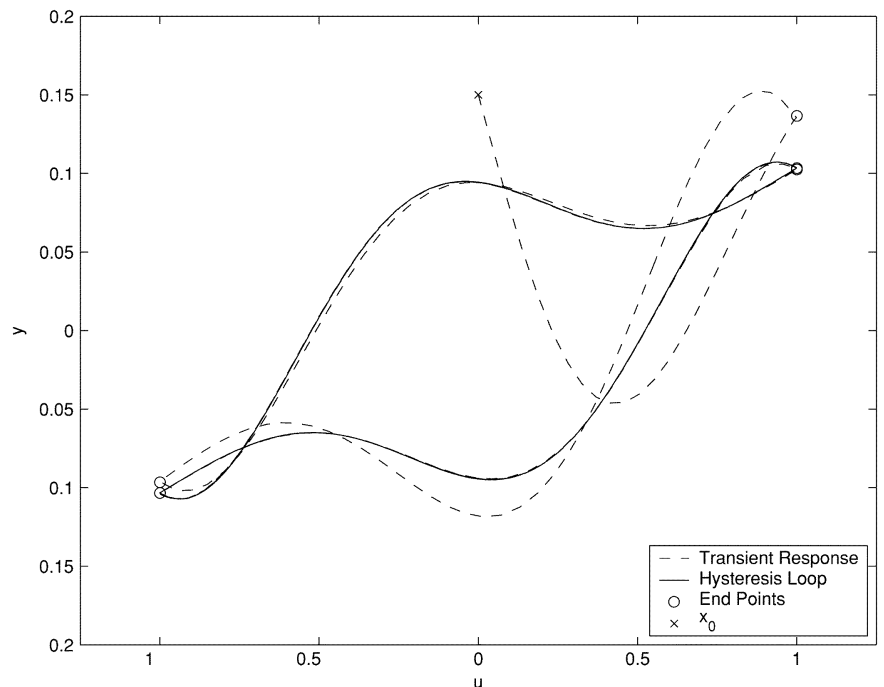

(a)

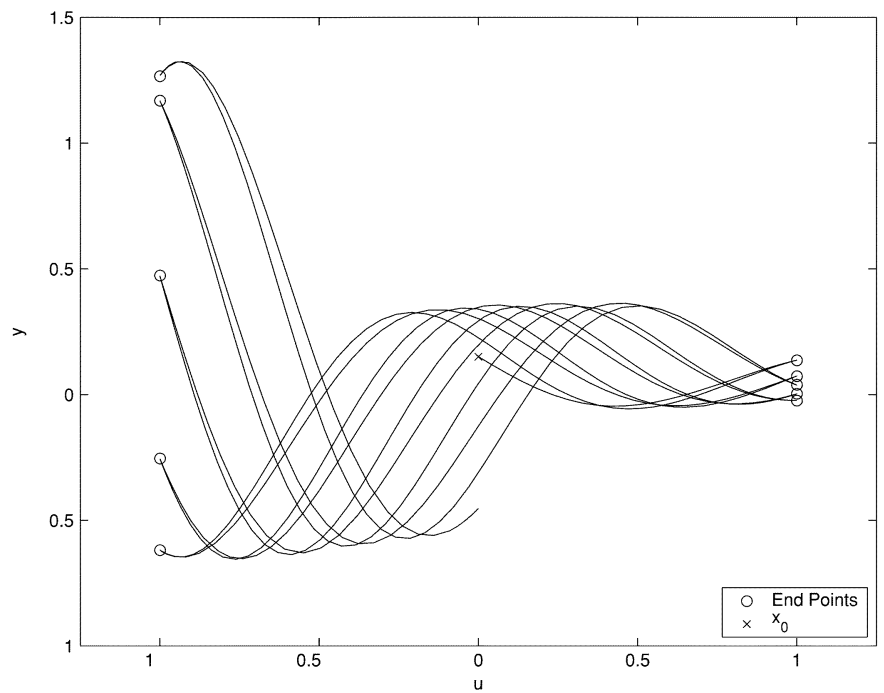

(b)

Fig. 12. Input-output maps of Example 4.2 under sinusoidal input with (a) $h_{+}=1$ and $h_{-}=-1$ and (b) $h_{+}=1$ and $h_{-}=1.1$.

For the following result, we assume that $g_{+}^{\prime}(0) \triangleq$ $\lim _{\alpha \downarrow 0}\left(g_{+}(\alpha)\right) /(\alpha)$ and $g_{-}^{\prime}(0) \triangleq \lim _{\alpha \uparrow 0}\left(g_{-}(\alpha)\right) /(\alpha)$ exist, where $\lim _{\alpha \uparrow 0}$ and $\lim _{\alpha \downarrow 0}$ denote the left- and right-hand limits at 0 , respectively.

Proposition 5.1: Consider the rate-dependent semilinear Duhem model (4.1) and (4.2), where $u:[0, \infty) \rightarrow\left[u_{\min }, u_{\max }\right]$ is $C^{1}$, periodic with period $\alpha$, and has exactly one local maximum $u_{\max }$ in $[0, \alpha)$ and exactly one local minimum $u_{\min }$ in $[0, \alpha)$. Furthermore, define $\beta \triangleq u_{\max }-u_{\min }$ and assume that $\rho\left(e^{\beta g_{+}^{\prime}(0) A_{+}} e^{-\beta g_{-}^{\prime}(0) A_{-}}\right)<1$. Then, the unique limiting periodic input-output map $\mathcal{H}_{\infty}(u)$ exists and is given by (4.7)-(4.14) with $A_{+}, A_{-}, B_{+}, B_{-}, E_{+}$and $E_{-}$replaced with $g_{+}^{\prime}(0) A_{+}, g_{-}^{\prime}(0) A_{-}, g_{+}^{\prime}(0) B_{+}, g_{-}^{\prime}(0) B_{-}, g_{+}^{\prime}(0) E_{+}$, and $g_{-}^{\prime}(0) E_{-}$, respectively.

Proof: Let $T>0$, let $u_{T}(t) \triangleq u(\alpha t / T)$, and consider the rate-independent semilinear Duhem model

$$
\begin{aligned}
& \Delta \dot{x}_{T}(t) \\
& \quad=g_{\Delta}\left(\dot{u}_{T}(t)\right)\left(\left[\begin{array}{l}
A_{+} \\
A_{-}
\end{array}\right] \Delta x_{T}(t)+\left[\begin{array}{l}
B_{+} \\
B_{-}
\end{array}\right] u_{T}(t)+\left[\begin{array}{l}
E_{+} \\
E_{-}
\end{array}\right]\right)
\end{aligned}
$$

$$
\begin{aligned}
& \Delta y_{T}(t) \\
& \quad=C \Delta x_{T}(t)+D u_{T}(t) \quad \Delta x_{T}(0)=x_{0}, \quad t \geq 0
\end{aligned}
$$

where

$$
g_{\Delta}\left(\dot{u}_{T}(t)\right) \triangleq\left[g_{+}^{\prime}(0) \dot{u}_{T_{+}}(t) I_{n} \quad g_{-}^{\prime}(0) \dot{u}_{T_{-}}(t) I_{n}\right]
$$

$\dot{u}_{T_{+}}(t) \triangleq \max \left\{0, \dot{u}_{T}(t)\right\}$, and $\dot{u}_{T_{-}}(t) \triangleq \min \left\{0, \dot{u}_{T}(t)\right\}$. Since (5.3), (5.4) is rate independent and since $\rho\left(e^{\beta g_{+}^{\prime}(0) A_{+}} e^{-\beta g_{-}^{\prime}(0) A_{-}}\right)<1$, Theorem 4.1 implies that the limiting periodic input-output map $\mathcal{H}_{\infty}(u)$ for $(5.3)$, (5.4) exists, $\Delta x_{T}$ is periodic, and the limiting input-output map $\mathcal{F}_{\infty}\left(u_{T}, \Delta y_{T}\right)$ is given by $\mathcal{H}_{\infty}(u)$. Our goal is to show that $\mathcal{H}_{\infty}(u)$ is the limiting input-output map for (5.1), (5.2). To do this we need to show that $\left\|y_{T}(t)-\Delta y_{T}(t)\right\| \rightarrow 0$ as $T \rightarrow \infty$ uniformly for all $t \geq 0$, so that the periodic input-output map of (5.1) and (5.2) converges to $\mathcal{H}_{\infty}(u)$ with respect to the Hausdorff metric (2.3).

For $T>0$, let $x_{T}(t) \in \mathbb{R}^{n}, t \geq 0$, satisfy (5.1), (5.2) with $u=u_{T}$ and $y=y_{T}$, define $g\left(\dot{u}_{T}(t)\right) \triangleq$ $\left[g_{+}\left(\dot{u}_{T}(t)\right) I_{n} \quad g_{-}\left(\dot{u}_{T}(t)\right) I_{n}\right]$, and define

$$
\begin{aligned}
p_{T}(t) \triangleq\left(g\left(\dot{u}_{T}(t)\right)-g_{\Delta}\left(\dot{u}_{T}(t)\right)\right. & \\
& \times\left(\left[\begin{array}{l}
A_{+} \\
A_{-}
\end{array}\right] \Delta x_{T}(t)+\left[\begin{array}{l}
B_{+} \\
B_{-}
\end{array}\right] u_{T}(t)+\left[\begin{array}{l}
E_{+} \\
E_{-}
\end{array}\right]\right) .
\end{aligned}
$$

Furthermore, define $e_{T}(t) \triangleq x_{T}(t)-\Delta x_{T}(t), t \geq 0$, which satisfies

$$
\begin{aligned}
\dot{e}_{T}(t) & =\dot{x}_{T}(t)-\Delta \dot{x}_{T}(t) \\
& =g\left(\dot{u}_{T}(t)\right)\left[\begin{array}{l}
A_{+} \\
A_{-}
\end{array}\right] e_{T}(t)+p_{T}(t)
\end{aligned}
$$

with $e_{T}(0)=0$. Let $t_{T, \max } \triangleq \min \left\{t \geq 0: u_{T}(t)=u_{\max }\right\}$ and $t_{T, \min } \triangleq \min \left\{t \geq 0: u_{T}(t)=u_{\min }\right\}$. For convenience, assume $t_{T, \max }<t_{T, \min }$. Then, for $q=0,1, \ldots$, (5.6) implies that $e_{T}(t)$ is given by (5.6), as shown at the bottom of the page, where $\Psi_{T_{+}}(t, \tau) \triangleq \exp \left[\int_{\tau}^{t} g\left(\dot{u}_{T}(\sigma)\right) A_{+} \mathrm{d} \sigma\right]$ and $\Psi_{T_{-}}(t, \tau) \triangleq$ $\exp \left[\int_{\tau}^{t} g\left(\dot{u}_{T}(\sigma)\right) A_{-} \mathrm{d} \sigma\right]$. Since $u$ is $C^{1}$ and thus $\dot{u}$ is bounded, it follows that $\lim _{T \rightarrow \infty} \dot{u}_{T}(t)=\lim _{T \rightarrow \infty}(\alpha / T) \dot{u}(\alpha t / T)=0$ uniformly for all $t \geq 0$. Furthermore, since $g$ is continuous at

$$
e_{T}(t)=\left\{\begin{array}{cc}
\int_{0}^{t} \Psi_{T_{+}}(t, \tau) p_{T}(\tau) \mathrm{d} \tau, & 0 \leq t \leq t_{T, \max } \\
\Psi_{T_{-}}\left(t, q T+t_{T, \max }\right) e_{T}\left(q T+t_{T, \max }\right) & \\
\quad+\int_{q T+t_{T, \text { max }}}^{t} \Psi_{T_{-}}(t, \tau) p_{T}(\tau) \mathrm{d} \tau, & q T+t_{T, \max }<t \leq q T+t_{T, \min } \\
\Psi_{T_{+}}\left(t, q T+t_{T, \min }\right) e_{T}\left(q T+t_{T, \min }\right) & \\
\quad+\int_{q T+t_{T, \text { min }}}^{t} \Psi_{T_{+}}(t, \tau) p_{T}(\tau) \mathrm{d} \tau, & q T+t_{T, \min }<t \leq(q+1) T+t_{T, \max }
\end{array}\right.
$$




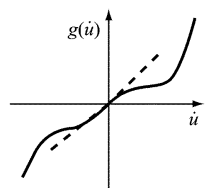

(a)

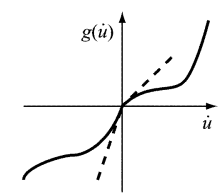

(b)

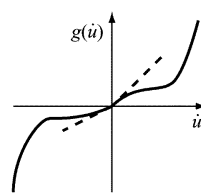

(c)

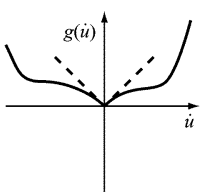

(d)
Fig. 13. Illustration of nonpositively homogeneous functions $g$ for asymptotically stable $A$. (a) Not hysteretic. (b) May not converge to $\mathcal{H}_{\infty}$ since $g_{-}^{\prime}(0)>g_{+}^{\prime}(0)$. (c), (d) Will converge to $\mathcal{H}_{\infty}$.

$0, \lim _{T \rightarrow \infty} g\left(\dot{u}_{T}(t)\right)=\lim _{u_{T} \rightarrow 0} g\left(\dot{u}_{T}\right)=0$ uniformly for all $t \geq 0$. Therefore

$$
\lim _{T \rightarrow \infty} \Psi_{T_{+}}(t, \tau)=\lim _{T \rightarrow \infty} \exp \left[\int_{\tau}^{t} g\left(\dot{u}_{T}(\sigma)\right) A_{+} \mathrm{d} \sigma\right]=I_{n}
$$

uniformly for all $t \geq 0$ and $\tau \in[0, t]$. Likewise, $\lim _{T \rightarrow \infty} \Psi_{T_{-}}(t, \tau)=I_{n}$ uniformly for all $t \geq 0$ and $\tau \in[0, t]$. Furthermore, since $g_{+}^{\prime}(0)$ and $g_{-}^{\prime}(0)$ exist

$$
\lim _{\dot{u}_{T} \downarrow 0}\left(g\left(\dot{u}_{T}\right)-g_{\Delta}\left(\dot{u}_{T}\right)\right)=\lim _{\dot{u}_{T} \uparrow 0}\left(g\left(\dot{u}_{T}\right)-g_{\Delta}\left(\dot{u}_{T}\right)\right)=0
$$

it follows that $\lim _{T \rightarrow \infty}\left(g\left(\dot{u}_{T}(t)\right)-g_{\Delta}\left(\dot{u}_{T}(t)\right)\right)=$ $\lim _{\dot{u}_{T} \rightarrow 0}\left(g\left(\dot{u}_{T}\right)-g_{\Delta}\left(\dot{u}_{T}\right)\right)=0$ uniformly for all $t \geq 0$. Therefore, since $\Delta x_{T}$ and $u_{T}$ are bounded, it follows that $\lim _{T \rightarrow \infty} p_{T}(t)=0$ uniformly for all $t \geq 0$ and, thus, $\lim _{T \rightarrow \infty} e_{T}(t)=0$ uniformly for all $t \geq 0$. Finally

$$
\begin{aligned}
\lim _{T \rightarrow \infty}\left\|y_{T}(t)-\Delta y_{T}(t)\right\| & =\lim _{T \rightarrow \infty}\left\|C x_{T}(t)-C \Delta x_{T}(t)\right\| \\
& \leq\|C\| \lim _{T \rightarrow \infty}\left\|e_{T}(t)\right\|=0 .
\end{aligned}
$$

As a special case of (5.1), (5.2), consider the rate-dependent semilinear Duhem model

$$
\begin{aligned}
& \dot{x}(t)=(A x(t)+B u(t)) g(\dot{u}(t)), \quad x(0)=x_{0}, t \geq 0 \\
& y(t)=C x(t)
\end{aligned}
$$

where $A \in \mathbb{R}^{n \times n}, B \in \mathbb{R}^{n}$, and $C \in \mathbb{R}^{1 \times n}$, and where $g$ is given by

$$
g(v)= \begin{cases}g_{+}(v), & v \geq 0 \\ g_{-}(v), & v<0\end{cases}
$$

The following result is the specialization of Proposition 5.1 to (5.7) and (5.8).

Corollary 5.1: Consider the rate-dependent semilinear Duhem model (5.1), (5.2) where $u:[0, \infty) \rightarrow\left[u_{\min }, u_{\max }\right]$ is $C^{1}$, and periodic with period $\alpha$ and has exactly one local maximum $u_{\max }$ in $[0, \alpha)$ and exactly one local minimum $u_{\min }$ in $[0, \alpha)$. Suppose that $A$ is asymptotically stable and assume $h_{-}<h_{+}$, where $h_{+} \triangleq g_{+}^{\prime}(0)$ and $h_{-} \triangleq g_{-}^{\prime}(0)$. Then, the unique limiting periodic input-output map $\mathcal{H}_{\infty}(u)$ exists and is given by (4.19)-(4.25).

Assuming that $A$ is asymptotically stable, Corollary $5.1 \mathrm{im}-$ plies that the existence of a limiting periodic input-output map for the rate-dependent semilinear Duhem model (5.7) and (5.8) depends on the slopes of $g$ at the origin. Fig. 13 illustrates several nonpositively homogeneous functions $g$. If $g_{+}^{\prime}(0)=g_{-}^{\prime}(0)$ as in Fig. 13(a), then the linearized rate-independent semilinear Duhem model is not hysteretic and, thus, (5.7) and (5.8) are not hysteretic. If $g_{+}^{\prime}(0) \neq g_{-}^{\prime}(0)$, it follows from Corollary 5.1 that for the functions $g$ shown in Fig. 13(c) and (d) the rate-dependent semilinear Duhem model has a limiting periodic input-output map.

\section{REVERSAL BEHAVIOR OF THE RATE-INDEPENDENT SEMILINEAR DUHEM MODEL}

In this section, we discuss the reversal behavior of the rate-independent semilinear Duhem model, that is, the change of the input-output map when the range of the input changes. Specifically, consider the rate-independent semilinear Duhem model (4.1), (4.2), where $u(t)$ is piecewise monotonic. Let $t_{1}>0$ and suppose that, for $0<t<t_{1}, u(t)$ is periodic with period $T$ and has exactly one local maximum $u_{\max }$ in $[0, T)$ and exactly one local minimum $u_{\min }$ in $[0, T)$. Let $\beta \triangleq u_{\max }-u_{\min }$ and suppose $\rho\left(e^{\beta A_{+}} e^{-\beta A_{-}}\right)<1$. Then, it follows from Theorem 4.1 that, for sufficiently large $t_{1}$, the input-output map of $u(t)$ and $y(t)$ converges to a limiting input-output map for $u \in\left[u_{\min }, u_{\max }\right]$. Now, let $u_{\min } \leq u_{\min _{1}}<u_{\max _{1}} \leq u_{\max }$, and suppose that for $t>t_{1}, u(t)$ is periodic with period $T_{1}$ and has exactly one local maximum $u_{\text {max }_{1}}$ in $\left[0, T_{1}\right)$ and exactly one local minimum $u_{\min _{1}}$ in $\left[0, T_{1}\right)$. Let $\beta_{1} \triangleq u_{\max _{1}}-u_{\min _{1}}$. If $\rho\left(e^{\beta_{1} A_{+}} e^{-\beta_{1} A_{-}}\right)<1$, then the input-output map of $u(t)$ and $y(t)$ converges to another limiting input-output map for $u \in\left[u_{\min _{1}}, u_{\max _{1}}\right]$ as $t \rightarrow \infty$. The limiting input-output map for $u \in\left[u_{\min }, u_{\max }\right]$ is the major loop and the limiting input-output map for $u \in\left[u_{\min _{1}}, u_{\max _{1}}\right]$ is a minor loop [2].

Note that if $A \in \mathbb{R}^{n \times n}$ is asymptotically stable, $B \in$ $\mathbb{R}^{n}, h_{-}<h_{+}$, and if $A_{+}=h_{+} A, A_{-}=h_{-} A, B_{+}=h_{+} B$, and $B_{-}=h_{-} B$, the existence of the minor loop is guaranteed for all $\beta_{2}>0$ from Corollary 4.1.

Example 6.1: Reconsider Example 4.2 with $h_{+}=1$ and $h_{-}=-1$. Let $u(t)=\sin t$ for $0 \leq t<10 \pi$, and $u(t)=$ $0.25 \sin t$ for $t \geq 10 \pi$. Since the range of the input changes from $[-1,1]$ to $[-0.25,0.25]$, we can determine the major and minor loops from (4.20) and (4.21). As shown in Fig. 14(a), the input-output map changes from the major loop to a minor loop after a transient response, as shown Fig. 14(b).

\section{ORIENTATION OF THE INPUT-OUTPUT MAP OF THE RATE-INDEPENDENT GENERALIZED DUHEM MODEL}

In this section, we analyze the orientation of the input-output map of the rate-independent generalized Duhem model (3.2) and (3.3). For the following discussion, we adopt standard terminology from complex analysis [25]. Let $\mathcal{H}$ be a closed curve, and let $\gamma:[0, \infty) \rightarrow \mathbb{R}^{2}$, where $\gamma(s)=\left(\gamma_{u}(s), \gamma_{y}(s)\right)$ be the periodic map with period $\alpha$ associated with $\mathcal{H}$ as defined in Definition 2.1. Then, $\mathcal{H}$ is simple if $\gamma(s) \neq \gamma(\hat{s})$ for all distinct $s, \hat{s} \in[0, \alpha)$. Furthermore, letting $\hat{\gamma}(s)=\gamma_{u}(s)+i \gamma_{y}(s)$, where $i \triangleq \sqrt{-1}$, then the winding number $n(\gamma, z)$ of $\gamma$ about $z=\left(z_{u}, z_{y}\right) \in \mathbb{R}^{2}$ is defined as

$$
n(\gamma, z)=\frac{1}{2 \pi i} \oint_{\hat{\gamma}} \frac{\mathrm{d} \zeta}{\zeta-\left(z_{u}+i z_{y}\right)} .
$$

Assume that $\mathcal{H}$ is a simple closed curve. Then it follows from the Jordan curve theorem $[25$, p. 111$]$ that $\mathbb{R}^{2} \backslash \mathcal{H}$ consists of two 


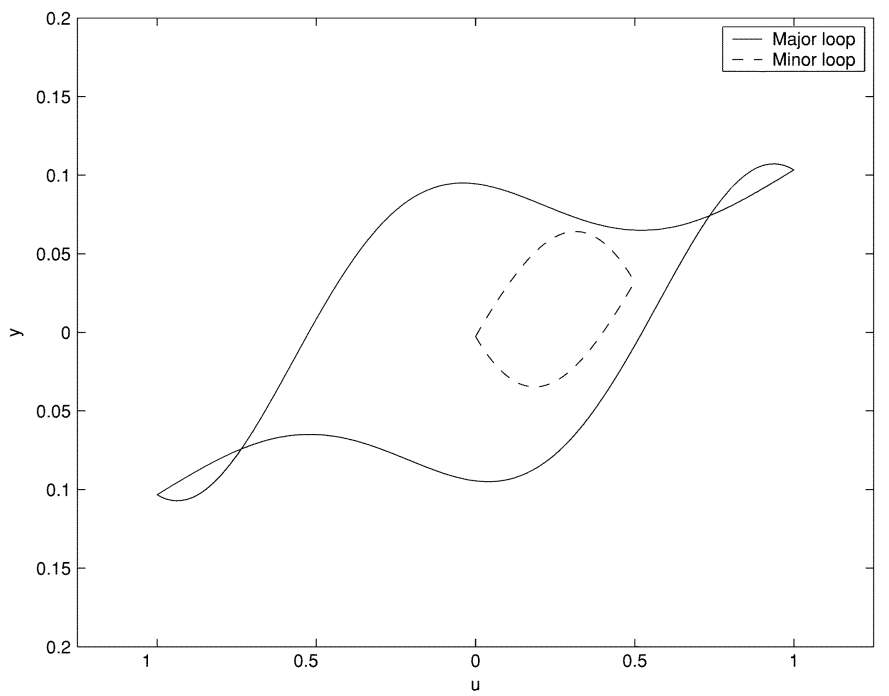

(a)

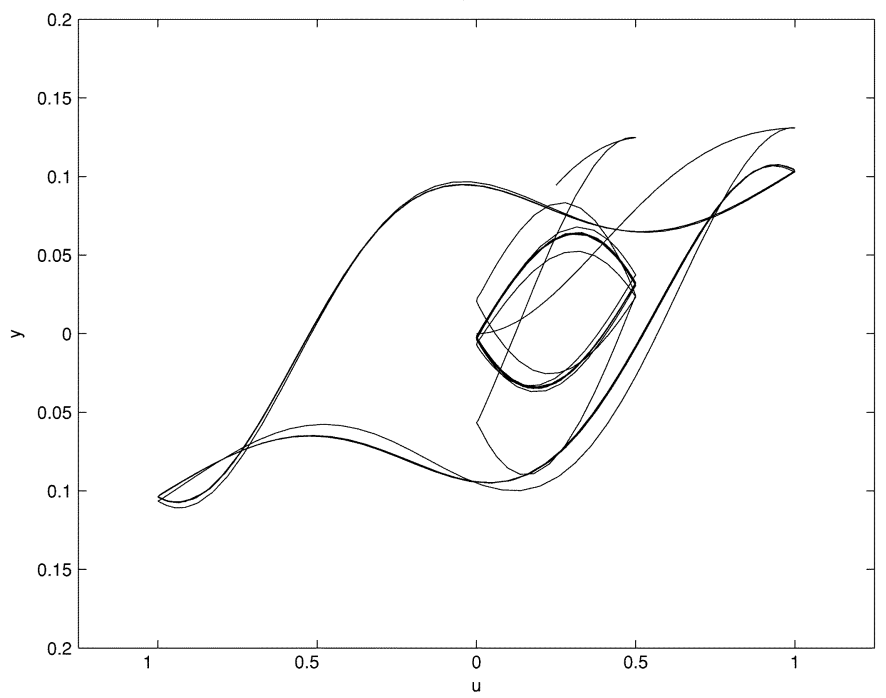

(b)

Fig. 14. (a) Major and minor loop of Example 6.1 from (4.20) and (4.21). (b) Transient response of Example 6.1 from the major loop to the minor loop.

disjoint connected open sets. The inside of the simple closed curve $\mathcal{H}$ is the bounded open set of these disjoint sets. Note that not every hysteretic limiting periodic input-output map $\mathcal{H}$ is a simple closed curve, since $\mathcal{H}$ can cross itself as shown in Fig. 12(a). The following definition is needed.

Definition 7.1: Let $u:[0, \infty) \rightarrow\left[u_{\min }, u_{\max }\right]$ be continuous, piecewise $C^{1}$, and periodic with period $\alpha$ and have exactly one local maximum $u_{\max }$ in $[0, \alpha)$ and exactly one local minimum $u_{\min }$ in $[0, \alpha)$. Let $y:[0, \infty) \rightarrow \mathbb{R}$ be periodic with period $\alpha$ and suppose that $u(t)$ and $y(t)$ satisfy the rate-independent generalized Duhem model (2.1), (2.2). Furthermore, define $\zeta(t) \triangleq(u(t), y(t))$ and assume that the limiting periodic input-output map $\mathcal{H}_{\infty}(u)=\zeta([0, \infty))(u)$ exists, and is hysteretic and simple. Then $\mathcal{H}_{\infty}(u)$ is clockwise (respectively, counterclockwise) if $n(\zeta, z)=1$ (respectively, $n(\zeta, z)=-1$ ) for every $z \in \mathbb{R}^{2}$ in the inside of $\mathcal{H}_{\infty}(u)$.

Let $u(t) \in\left[u_{\min }, u_{\max }\right]$ be piecewise monotonic and consider the reparameterized rate-independent generalized Duhem model (3.8), (3.9). Assume that the limiting periodic
CW:
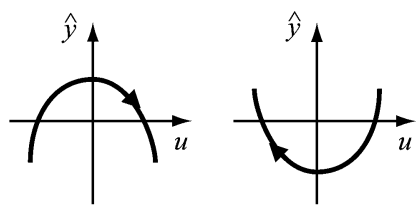

$$
\dot{u}>0, \frac{\mathrm{d} 2 \hat{y}}{\mathrm{~d} u^{2}}<0 \quad \dot{u}<0, \frac{\mathrm{d} 2 \hat{y}}{\mathrm{~d} u^{2}}>0
$$

CCW:
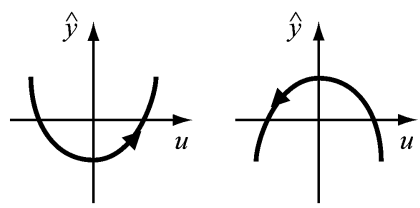

$$
\dot{u}>0, \frac{\mathrm{d} 2 \hat{y}}{\mathrm{~d} u^{2}}>0 \quad \dot{u}<0, \frac{\mathrm{d} 2 \hat{y}}{\mathrm{~d} u^{2}}<0
$$

Fig. 15. Relationship between clockwise orientation (CW) and counterclockwise orientation $(\mathrm{CCW})$ of the input-output map and the sign of $\left(\mathrm{d}^{2} \hat{y}(u)\right) /\left(\mathrm{d} u^{2}\right)$.

input-output map $\mathcal{H}_{\infty}(u)$ exists, and is hysteretic and simple. Now, suppose the positive orbit of (3.8), (3.9) is concave and the negative orbit of (3.8), (3.9) is convex or, equivalently

$$
\frac{\mathrm{d}^{2} \hat{y}(u)}{\mathrm{d} u^{2}}\left\{\begin{array}{ll}
<0, & \text { when } u \text { increases, } \\
>0, & \text { when } u \text { decreases, }
\end{array} \quad u \in\left[u_{\min }, u_{\max }\right]\right.
$$

which implies $\mathcal{H}_{\infty}(u)$ is clockwise. Similarly, suppose the positive orbit of (3.8), (3.9) is convex and the negative orbit of (3.8), (3.9) is concave, or equivalently

$$
\frac{\mathrm{d}^{2} \hat{y}(u)}{\mathrm{d} u^{2}} \begin{cases}>0, & \text { when } u \text { increases, } \\ <0, & \text { when } u \text { decreases, }\end{cases}
$$

which implies that $\mathcal{H}_{\infty}(u)$ is counterclockwise. Fig. 15 shows the relationship between the orientation of the trajectory and the signs of $\left(\mathrm{d}^{2} \hat{y}(u)\right) /\left(\mathrm{d} u^{2}\right)$. This observation shows that the orientation of the limiting periodic input-output map of the rate-independent generalized Duhem model can be determined by the concavity and convexity of $\hat{y}(u)$, or by the sign of the second derivative of $\hat{y}(u)$ with respect to $u$. We thus obtain the following result for the input-output map of the rate-independent generalized Duhem model.

Proposition 7.1: Let $u:[0, \infty) \rightarrow\left[u_{\min }, u_{\max }\right]$ be continuous, piecewise $C^{1}$, and periodic with period $\alpha$ and have exactly one local maximum $u_{\max }$ in $[0, \alpha)$ and exactly one local minimum $u_{\min }$ in $[0, \alpha)$. Consider the rate-independent generalized Duhem model (3.2), (3.3). Assume that $h$ is $C^{2}$ with respect to $x$ and $u$. Also assume that the limiting periodic input-output map $\mathcal{H}_{\infty}(u)$ exists, and is hysteretic and simple. Furthermore, for $u \in\left[u_{\min }, u_{\max }\right]$, define

$$
\begin{aligned}
\mathcal{O}_{+} & (u) \\
& \triangleq f_{+}^{\mathrm{T}}(\hat{x}(u), u) h_{x x}(\hat{x}(u), u) f_{+}(\hat{x}(u), u) \\
& +h_{x u}(\hat{x}(u), u) f_{+}(\hat{x}(u), u) \\
& +h_{x}(\hat{x}(u), u) f_{+_{x}}(\hat{x}(u), u) f_{+}(\hat{x}(u), u) \\
& +h_{x}(\hat{x}(u), u) f_{+u}(\hat{x}(u), u)+h_{u x}(\hat{x}(u), u) f_{+}(\hat{x}(u), u) \\
& +h_{u u}(\hat{x}(u), u)
\end{aligned}
$$




$$
\begin{aligned}
& \mathcal{O}_{-}(u) \\
& \quad \triangleq f_{-}^{\mathrm{T}}(\hat{x}(u), u) h_{x x}(\hat{x}(u), u) f_{-}(\hat{x}(u), u) \\
& \quad+h_{x u}(\hat{x}(u), u) f_{-}(\hat{x}(u), u) \\
& \quad+h_{x}(\hat{x}(u), u) f_{-x}(\hat{x}(u), u) f_{-}(\hat{x}(u), u) \\
& \quad+h_{x}(\hat{x}(u), u) f_{-u}(\hat{x}(u), u)+h_{u x}(\hat{x}(u), u) f_{-}(\hat{x}(u), u) \\
& \quad+h_{u u}(\hat{x}(u), u) .
\end{aligned}
$$

If $\mathcal{O}_{+}(u)<0$ and $\mathcal{O}_{-}(u)>0$ for all $u \in\left[u_{\min }, u_{\text {max }}\right]$, then $\mathcal{H}_{\infty}(u)$ is clockwise. Similarly, if $\mathcal{O}_{+}(u)>0$ and $\mathcal{O}_{-}(u)<0$ for all $u \in\left[u_{\min }, u_{\max }\right]$, then $\mathcal{H}_{\infty}(u)$ is counterclockwise.

Proof: For $u \in\left[u_{\min }, u_{\max }\right]$, it suffices to show that $\left(\mathrm{d}^{2} \hat{y}(u)\right) /\left(\mathrm{d} u^{2}\right)=\mathcal{O}_{+}(u)$ when $u$ increases, and $\left(\mathrm{d}^{2} \hat{y}(u)\right) /\left(\mathrm{d} u^{2}\right)=\mathcal{O}_{-}(u)$ when $u$ decreases. Suppose $u$ is increasing. Then

$$
\begin{aligned}
\frac{\mathrm{d}^{2} \hat{y}}{\mathrm{~d} u^{2}}(u)= & \frac{\mathrm{d}}{\mathrm{du}}\left(\frac{\mathrm{d}}{\mathrm{du}} h(\hat{x}(u), u)\right) \\
= & \frac{\mathrm{d}}{\mathrm{du}}\left(h_{x}(\hat{x}(u), u) f_{+}(\hat{x}(u), u)+h_{u}(\hat{x}(u), u)\right) \\
= & \frac{\mathrm{d}}{\mathrm{du}} h_{x}(\hat{x}(u), u) f_{+}(\hat{x}(u), u) \\
& +h_{x}(\hat{x}(u), u) \frac{\mathrm{d}}{\mathrm{du}} f_{+}(\hat{x}(u), u)+\frac{\mathrm{d}}{\mathrm{du}} h_{u}(\hat{x}(u), u) \\
= & f_{+}^{\mathrm{T}}(\hat{x}(u), u) h_{x x}(\hat{x}(u), u) f_{+}(\hat{x}(u), u) \\
& +h_{x u}(\hat{x}(u), u) f_{+}(\hat{x}(u), u) \\
& +h_{x}(\hat{x}(u), u) f_{+_{x}}(\hat{x}(u), u) f_{+}(\hat{x}(t), u) \\
& +h_{x}(\hat{x}(u), u) f_{+u}(\hat{x}(u), u) \\
& +h_{u x}(\hat{x}(u), u) f_{+}(\hat{x}(t), u)+h_{u u}(\hat{x}(u), u) .
\end{aligned}
$$

The proof is similar for decreasing $u$.

Remark 7.1: For the semilinear Duhem model (4.1), (4.2), $\mathcal{O}_{+}$and $\mathcal{O}_{-}$are given by

$$
\begin{aligned}
& \mathcal{O}_{+}(u)=C A_{+}^{2} \hat{x}(u)+C A_{+} B_{+} u+C B_{+}+C A_{+} E_{+}(7.4) \\
& \mathcal{O}_{-}(u)=C A_{-}^{2} \hat{x}(u)+C A_{-} B_{-} u+C B_{-}+C A_{-} E_{-}(.7 .5)
\end{aligned}
$$

Example 7.1: Consider the generalized Duhem model

$$
\begin{aligned}
& \dot{x}(t)=(-x(t)+b u(t))|u(t)| \quad x(0)=0, \quad t \geq 0 \\
& y(t)=x(t) .
\end{aligned}
$$

Let $u(t)=\sin t, t \geq 0$. Then Corollary 4.1 implies that the limiting periodic input-output map $\mathcal{H}_{\infty}(u)$ exists for all $b \in \mathbb{R}$. First, let $b=1$. Then, it can be seen that $\mathcal{O}_{+}(u)>0$ and $\mathcal{O}_{-}(u)<0$ for all $u \in[-1,1]$. Hence, it follows from Proposition 7.1 that $\mathcal{H}_{\infty}(u)$ is counterclockwise, as shown in Fig. 16(a). Now, let $b=-1$. Then, it can be seen that $\mathcal{O}_{+}(u)<0$ and $\mathcal{O}_{-}(u)>0$ for all $u \in[-1,1]$. Hence, it follows from Proposition 7.1 that $\mathcal{H}_{\infty}(u)$ is clockwise, as shown in Fig. 16(b).

\section{CONCLUSION}

In this paper, we introduced the rate-independent and rate-dependent semilinear Duhem model. The analysis of the rate-independent model was facilitated by a reparameterization in terms of the control input. By analyzing the iterated ramp response,

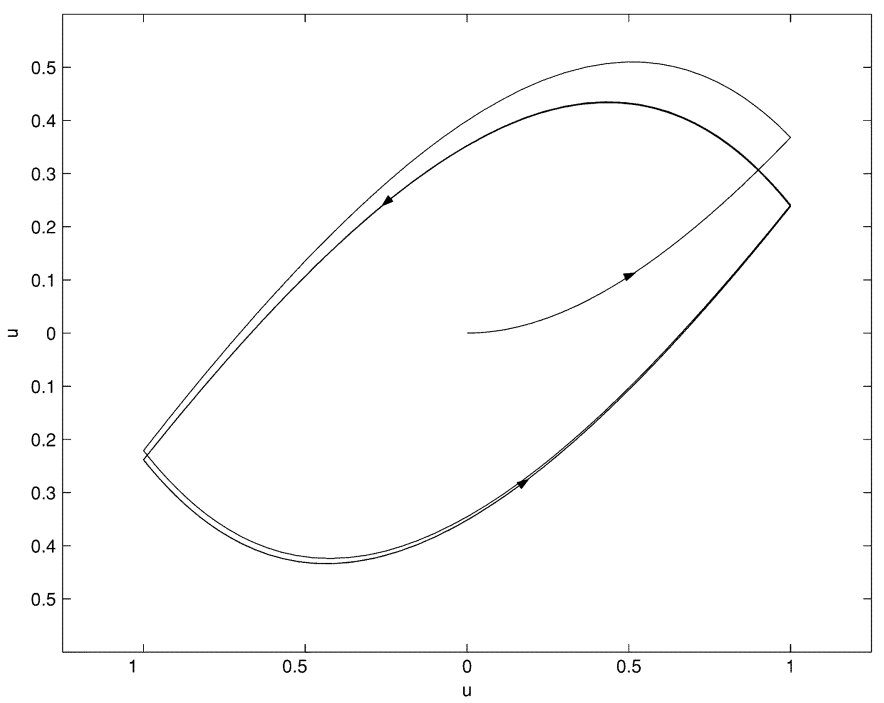

(a)

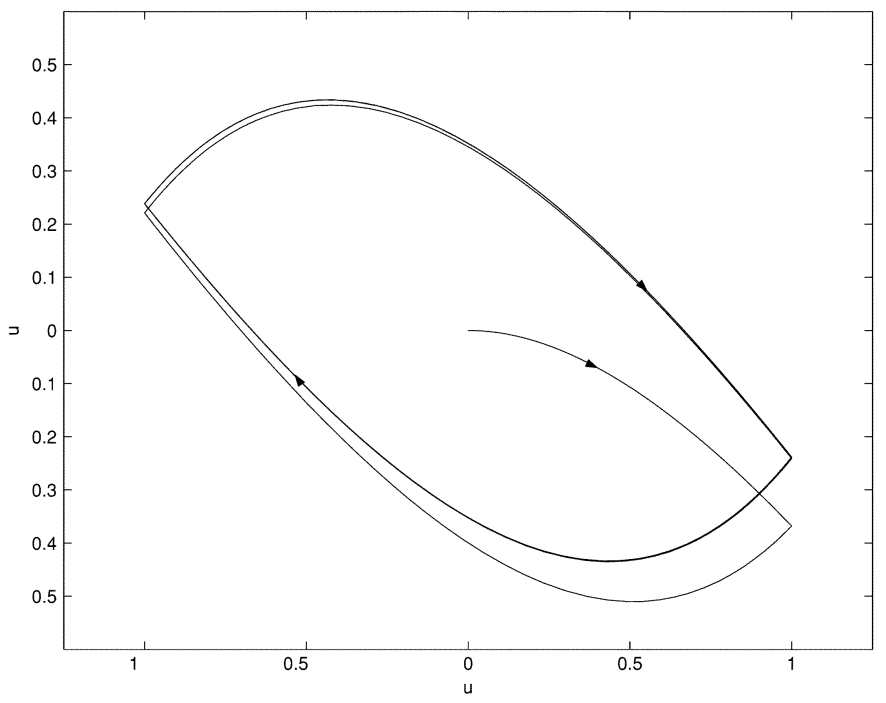

(b)

Fig. 16.Input-output map of Example 7.1 with (a) $b=1$ and (b) $b=-1$. The orientation of the input-output map is counterclockwise when $b=1$, and clockwise when $b=-1$.

we obtained sufficient conditions for convergence to a limiting input-output map. We then approximated the rate-dependent model with low frequency inputs by a rate-independent semilinear Duhem model and developed a sufficient condition for convergence. We then analyzed the orientation of the rate-independent hysteresis map in terms of its clockwise or counterclockwise evolution. The reversal behavior of the rate-independent semilinear Duhem model was characterized by applying these results to a restricted input range. Future research possibilities include developing conditions for the existence of hysteresis for the generalized Duhem model.

\section{REFERENCES}

[1] S. L. Lacy, D. S. Bernstein, and S. P. Bhat, "Hysteretic systems and step-convergent semistability," in Proc. Amer. Control Conf., Chicago, IL, Jun. 2000, pp. 4139-4143. 
[2] B. D. Coleman and M. L. Hodgdon, "A constitutive relation for rateindependent hysteresis in ferromagnetically soft materials," Int. J. Eng. Sci., vol. 24, pp. 897-919, 1986.

[3] M. L. Hodgdon, "Applications of a theory of ferromagnetic hysteresis," IEEE Trans. Magn., vol. 24, no. 1, pp. 218-221, Jan. 1988.

[4] J. W. Macki, P. Nistri, and P. Zecca, "Mathematical models for hysteresis," SIAM Rev., vol. 35, no. 1, pp. 94-123, 1993.

[5] I. D. Mayergoyz, "Dynamic Preisach models of hysteresis," IEEE Trans. Magn., vol. 24, no. 6, pp. 2925-2927, Jun. 1988.

[6] M. Brokate and A. Visintin, "Properties of the Preisach model for hysteresis," J. Reineund Angewandte Mathematik, vol. 402, pp. 1-40, 1989.

[7] I. D. Mayergoyz, Mathematical Models of Hysteresis. New York: Springer-Verlag, 1991.

[8] R. B. Gorbet, K. A. Morris, and D. W. L. Wang, "Control of hysteretic systems: A state space approach," in Learning, Control and Hybrid Systems. New York, 1998, pp. 432-451.

[9] W. Haddad, V. Chellaboina, and J. Oh, "Linear controller analysis and design for systems with input hystereses nonlinearites," J. Franklin Inst., vol. 340, pp. 371-390, 2003.

[10] F. T. Calkins, R. C. Smith, and A. B. Flatau, "Energy-based hysteresis model for magnetostrictive transducers," IEEE Trans. Magn., vol. 36, no. 2, pp. 429-439, Mar. 2000.

[11] V. Lampaert and J. Swevers, "On-line identification of hysteresis functions with nonlocal memory," in IEEE/ASME Int. Conf. Advanced Intelligent Mechatronics, Como, Italy, 2001, pp. 833-837.

[12] D. D. Rizos and S. D. Fassois, "Presliding friction identification based upon the Maxwell slip model structure," Chaos, vol. 14, no. 2, pp. 431-445, 2004.

[13] M. A. Krasnosel'skii and A. V. Pokrovskii, Systems with Hysteresis. New York: Springer-Verlag, 1980.

[14] L. O. Chua and S. C. Bass, "A generalized hysteresis model," IEEE Trans. Circuit Theory, vol. 19, no. 1, pp. 36-48, Jan. 1972.

[15] A. Visintin, Differential Models of Hysteresis. New York: SpringerVerlag, 1994.

[16] R. Banning, W. L. Koning, H. J. Adriaens, and R. K. Koops, "State-space analysis and identification for a class of hysteretic systems," Automatica, vol. 37, pp. 1883-1892, 2001.

[17] R. Bouc, "Modèle mathématique d'hystérésis," Acustica, vol. 24, pp. 16-25, 1971 .

[18] Y. K. Wen, "Method for random vibration of hysteretic systems," in $J$. Eng. Mech. Division, Proc. ASCE, vol. 102, 1976, pp. 249-263.

[19] P. Dahl, "Solid friction damping of mechanical vibrations," in AIAA J., vol. 14,1976 , pp. 1675-82.

[20] C. C. de Wit, H. Olsson, K. J. Astrom, and P. Lischinsky, "A new model for control of systems with friction," IEEE Trans. Autom. Control, vol. 40, no. 3, pp. 419-425, Mar. 1995.
[21] J. Swevers, F. Al-Bender, C. G. Ganseman, and T. Prajogo, "An integrated friction model structure with improved presliding behavior for accurate friction compensation," IEEE Trans. Autom. Control, vol. 45, no. 4, pp. 675-686, Apr. 2000.

[22] C. D. Aliprantis and K. C. Border, Infinite Dimensional Analysis: A Hitchhiker's Guide, 2nd ed. Berlin, Germany: Springer-Verlag, 1999.

[23] G. A. Edgar, Measure, Topology, and Fractal Geometry. New York: Springer-Verlag, 1990.

[24] S. L. Campbell and J. C. D. Meyer, Generalized Inverse of Linear Transformations. London, U.K.: Pitman, 1979.

[25] B. P. Palka, An Introduction to Complex Function Theory. New York: Springer-Verlag, 1990.

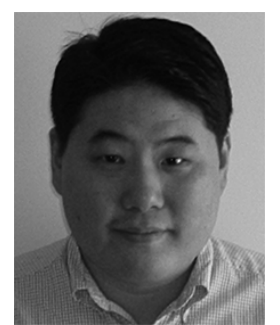

JinHyoung Oh (S'02) was born in Taegu, South Korea, in 1971. He received the B.S. degree in control and instrumentation engineering from Korea University, Chochiwon, Korea, in 1993, and the M.S. degrees in aerospace engineering and applied mathematics from the Georgia Institute of Technology, Atlanta, in 1999 and 2001, respectively. He will receive the $\mathrm{Ph} . \mathrm{D}$. degree in aerospace engineering from the University of Michigan, Ann Arbor, in April 2005. His Ph.D. thesis focuses on modeling, identification, and control of hysteresis.

He is currently a Research Fellow with the University of Michigan.

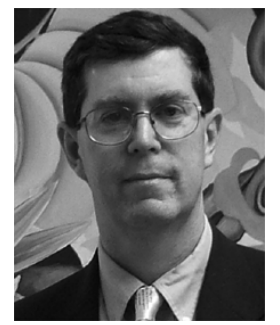

Dennis S. Bernstein (M'82-SM'99-F'01) received the Sc.B. degree in applied mathematics from Brown University, Providence, RI, in 1977, and the Ph.D. degree in control engineering from the University of Michigan, Ann Arbor, in 1982.

$\mathrm{He}$ is currently a Professor in the Aerospace Engineering Department at the University of Michigan. His interests encompass all aspects of control for aerospace applications, with emphasis on identification and adaptive control for vibration and flow control. He is the author of Matrix Mathematics: Theory, Facts, and Formulas with Application to Linear Systems Theory (Princeton, NJ: Princeton Univ. Press, 2004). 